\title{
Master Equation Study of Hydrogen Relaxation Using Complete Sets of State-to-state Transition Rates
}

\author{
Jae Gang Kim ${ }^{1}$ and Iain D. Boyd ${ }^{2}$ \\ University of Michigan, Ann Arbor, MI 48109-2140
}

\begin{abstract}
The complete sets of state-to-state transition rate coefficients for both target and projectile molecules are derived from the predicted response surface designed by the ordinary Kriging model. A system of master equations is constructed for bound-bound and bound-free transitions with these designed transition rate coefficients, and the rovibrational number densities are numerically evaluated by implicitly integrating a system of master equations. In these master equation studies, relaxation of rotation and vibration modes, number density relaxation, reaction rate coefficients, and average rotational and vibrational energy losses due to dissociation are each considered in strong nonequilibrium conditions. A system of master equations are coupled with one-dimensional flow equations to analyze the relaxations of $\mathrm{H}_{2}$ in post-normal shock and nozzle expanding flows. In post-normal shock flows, the relaxation of the rotational mode is slightly faster or almost similar to the relaxation of the vibrational mode. In nozzle expanding flows, the relaxations of both rotational and vibrational modes seem to be frozen.
\end{abstract}

\section{Nomenclature}

$\begin{array}{ll}A & \text { area, } \mathrm{cm}^{2} \\ c & \text { dissociation of molecule } \\ D_{i} & \text { dissociation energy of rovibrational state } i \text {, erg } \\ e_{i} & \text { rovibrational energy of state } i, \text { erg } \\ e_{r}, e_{v} & \text { averaged rotational and vibrational energies, respectively, erg } \\ e_{r}(i), e_{v}(i) & \text { rotational and vibrational energies of rovibrational state } i \text {, respectively, erg } \\ e_{r}, e_{v} & \text { averaged rotational and vibrational energy losses, respectively, erg } \\ E_{t r} & \text { relative translational energy, erg } \\ h & \text { enthalpy, erg/g } \\ h^{f} & \text { formation energy, erg/mol } \\ g_{i j \ell m}, g_{i j \ell}, g_{i j m}, g_{i j} & \text { parameter to prohibit the multiple count } \\ g_{e} & \text { statistical multiplicity of electronic state } \\ g_{s} & \text { nuclear spin degeneracy } \\ K & \text { state-to-state transition rate coefficient, } \mathrm{cm}^{3} \mathrm{sec}^{-1} \\ K_{f} & \text { dissociation reaction rate coefficients, } \mathrm{cm}^{3} \mathrm{sec}^{-1} \\ K_{f-\text { oneway }} & \text { one-way dissociation reaction rate coefficients, } \mathrm{cm}^{3} \mathrm{sec}^{-1} \\ K_{r} & \text { recombination reaction rate coefficients, } \mathrm{cm}^{6} \mathrm{sec}^{-1} \\ m_{r} & \text { reduced mass, } g \\ n_{i} & \text { number density of rovibrational state } i, \mathrm{~cm}^{-3} \\ n_{H} & \text { number density of hydrogen atomic species, } \mathrm{cm}^{-3} \\ N_{A} & \text { Avogadro number, } \text { mol }^{-1} \\ & \end{array}$

\footnotetext{
${ }^{1}$ Post-doctoral Research Fellow, Department of Aerospace Engineering, jaegkim@umich.edu, Member AIAA.

${ }^{2}$ Professor, Department of Aerospace Engineering, iainboyd@umich.edu, Fellow AIAA.
}

American Institute of Aeronautics and Astronautics 


\begin{tabular}{|c|c|}
\hline$p$ & pressure, dyne/ $\mathrm{cm}^{2}$ \\
\hline$Q_{H}$ & atomic partition function of hydrogen atomic species \\
\hline$Q_{i}$ & partition function of rovibrational state $i$ \\
\hline$Q_{m}$ & molecular partition function \\
\hline$Q_{t}$ & translational partition function \\
\hline$u$ & flow velocity, $\mathrm{cm} / \mathrm{sec}$ \\
\hline$t$ & time, sec \\
\hline$T, T_{r}, T_{v}$ & translational, rotational, and vibrational temperatures, respectively, $K$ \\
\hline$\left(v_{1}, j_{1}\right),\left(v_{1}^{\prime}, j_{1}^{\prime}\right)$ & initial and final rovibrational states of a target molecule \\
\hline$\left(v_{2}, j_{2}\right),\left(v_{2}^{\prime}, j_{2}^{\prime}\right)$ & initial and final rovibrational states of a projectile molecule \\
\hline$w$ & relative speed, $\mathrm{cm} / \mathrm{sec}$ \\
\hline$x$ & distance, $\mathrm{cm}$ \\
\hline$\varepsilon$ & symmetric factor of homo- or hetero-geneous molecule \\
\hline$\varphi_{i}$ & normalized population of rovibrational state $i$ \\
\hline$\gamma_{i}$ & rovibrational concentration of state $i, \mathrm{~mol} / \mathrm{g}$ \\
\hline$\gamma_{H}, \gamma_{H e}$ & species concentration of hydrogen atom and helium species, $\mathrm{mol} / \mathrm{g}$ \\
\hline$\rho$ & density, $\mathrm{g} / \mathrm{cm}^{3}$ \\
\hline$\sigma$ & state-to-state transition cross section, $\mathrm{cm}^{2}$ \\
\hline \multicolumn{2}{|l|}{ Subscription } \\
\hline$E q$ & equilibrium state \\
\hline exit & nozzle exit \\
\hline$s$ & settling chamber \\
\hline th & nozzle throat \\
\hline$\infty$ & free stream \\
\hline
\end{tabular}

\section{Introduction}

$\mathrm{T}$

$\mathrm{HE}$ atmospheres of outer planets consist mostly of $\mathrm{H}_{2}$ and small concentrations of $\mathrm{He}$ and $\mathrm{CH}_{4}$. For future outer planetary missions, it is necessary to analyze the nonequilibrium phenomena of $\mathrm{H}_{2}$. For the entry flight into Jupiter, the shock layer flow can be considered to be in thermochemical equilibrium because of the relatively high stagnation pressures. In the flights into other outer planets, peak heating is likely to occur at lower stagnation pressures, and therefore, the shock layer flow may not be in equilibrium. In previous works ${ }^{1,2}$ the thermochemical nonequilibrium for $\mathrm{H}, \mathrm{He}$ and $\mathrm{H}_{2}$ mixtures were studied. Nonequilibrium excitation processes of rotational and vibrational modes of the electronic ground state were calculated accurately through state-to-state transition rate coefficients and master equations. The quasi-classical trajectory (QCT) method ${ }^{3,4}$ was adopted to calculate the stateto-state transition cross sections and rate coefficients for 348 rovibrational states of $\mathrm{H}_{2}$. In the work ${ }^{2}$ for $\mathrm{H}_{2}+\mathrm{H}_{2}$ collisions, the internal states of the projectile molecule were assumed to be distributed in a Boltzmann distribution specified by a nonequilibrium temperature. The nonequilibrium temperature was in turn determined from the rovibrational energy content. This assumption was adopted to overcome the difficulties of computational resources. However, it has a limitation; In a faithful analysis of rotation-to-translation, vibration-to-translation, and rotation-tovibration energy transitions, the state-to-state transitions of internal states of the target and projectile molecules must be described. However, in this assumption, the state-to-state transitions of internal states of a projectile molecule cannot be described. In describing the energy transitions for both target and projectile molecules, complete sets of the state-to-state transition rate coefficients of both molecules are needed. There are 348 rovibrational states in $\mathrm{H}_{2}$. Therefore, 60,726 different types of collision pairs between the target and projectile molecules are necessary to make the transition rate coefficients for each translational temperature. Evaluating these transition rate coefficients requires prohibitively large computing resources.

In the present work, the complete sets of the state-to-state transition rate coefficients for the 60,726 initial collision pairs are derived from the state-to-state transition cross sections of a smaller number of initial collision pairs between the target and projectile molecules. Specific rovibrational energy states are randomly selected in the 
348 rovibrational energy states. For these selected energy states, a smaller number of initial collision pairs are constructed. The state-to-state transition cross sections for these initial collision pairs are evaluated by QCT calculations for various relative translational energies from $0.1 \mathrm{eV}$ to $6 \mathrm{eV}$. By using these cross sections, the response surfaces are designed by the Kriging model. ${ }^{5-7}$ From the designed response surfaces, complete sets of the state-to-state transition cross sections are derived. The transition rate coefficients for the 60,726 initial collision pairs are calculated by an integration of these cross sections over the relative velocity between the colliding partners. A system of the master equation is constructed with these rate coefficients. From the results of the master equation calculation, the relaxation rate of the vibrational and rotational modes, the relaxation rate of species number density, the quasi-steady state (QSS) reaction rate coefficients, and the average rovibrational energy transferred during dissociation and recombination are derived. One-dimensional nozzle and post-normal shock flows of $\mathrm{H}_{2}$ and $\mathrm{He}$ mixtures are also calculated by coupling the flow and master equations.

\section{State-to-state transition rate coefficients}

In the present work, a master equation study is performed by using complete sets of state-to-state transition rate coefficients including the rovibrational energy transitions not only for the target, but also for the projectile molecules. In these rate coefficients, the transition processes are:

$$
\begin{array}{ll}
H_{2}\left(v_{1}, j_{1}\right)+H_{2}\left(v_{2}, j_{2}\right) \square \quad H_{2}\left(v_{1}{ }^{\prime}, j_{1}{ }^{\prime}\right)+H_{2}\left(v_{2}{ }^{\prime}, j_{2}{ }^{\prime}\right) \\
H_{2}\left(v_{1}, j_{1}\right)+H_{2}\left(v_{2}, j_{2}\right) \square \quad H_{2}\left(v_{1}{ }^{\prime}, j_{1}{ }^{\prime}\right)+2 H \\
H_{2}\left(v_{1}, j_{1}\right)+H_{2}\left(v_{2}, j_{2}\right) \square & 2 H+H_{2}\left(v_{2}{ }^{\prime}, j_{2}{ }^{\prime}\right) \\
H_{2}\left(v_{1}, j_{1}\right)+H_{2}\left(v_{2}, j_{2}\right) \square & 4 H
\end{array} .
$$

The bound-bound and bound-free transitions are defined as

$$
\begin{aligned}
& \sigma\left(v_{1}, j_{1}, v_{2}, j_{2} \rightarrow v_{1}{ }^{\prime}, j_{1}{ }^{\prime}, v_{2}{ }^{\prime}, j_{2}{ }^{\prime} ; E_{t r}\right) \\
& K\left(v_{1}, j_{1}, v_{2}, j_{2} \rightarrow v_{1}{ }^{\prime}, j_{1}{ }^{\prime}, v_{2}{ }^{\prime}, j_{2}{ }^{\prime} ; T_{t r}\right)=4 \pi \int_{E_{0} \times(}^{\infty}\left(\frac{m_{r}}{2 \pi k T}\right) \exp \left(\frac{m_{r} w^{2}}{2 k T}\right) w^{3} d w, \\
& \sigma\left(v_{1}, j_{1}, v_{2}, j_{2} \rightarrow v_{1}{ }^{\prime}, j_{1}{ }^{\prime}, c ; E_{t r}\right) \\
& K\left(v_{1}, j_{1}, v_{2}, j_{2} \rightarrow v_{1}{ }^{\prime}, j_{1}{ }^{\prime}, c ; T_{t r}\right)=4 \pi \int_{E_{0} \times}^{\infty}\left(\frac{m_{r}}{2 \pi k T}\right) \exp \left(\frac{m_{r} w^{2}}{2 k T}\right) w^{3} d w \quad, \\
& \sigma\left(v_{1}, j_{1}, v_{2}, j_{2} \rightarrow c, v_{2}{ }^{\prime}, j_{2}{ }^{\prime} ; E_{t r}\right)
\end{aligned}
$$

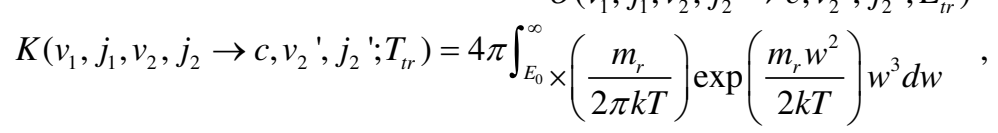

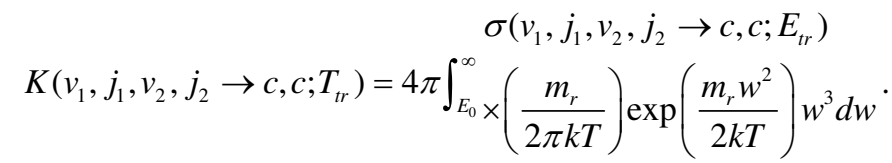

There are 60,726 initial collision pairs between the target and projectile molecules to make these transition rate coefficients for each temperature. In the present work, the transition rate coefficients of 60,726 initial collision pairs are derived from the state-to-state transition cross sections of a smaller number of initial collision pairs between the target and projectile molecules by using the Kriging model. ${ }^{5-7}$

The Kriging model $^{5}$ was originally developed to analyze the results of physical experiments to create empirically based models of the observed response values. This model was applied in the field of deterministic simulation for the design and analysis of computer experiments by Sacks et al. ${ }^{6}$ In the present work, the ordinary Kriging model ${ }^{7}$ is adopted to design a response surface of state-to-state transition cross sections. The ordinary Kriging model treats the deterministic response $y(\underline{x})$ as a realization of a stochastic process that can be expressed in a regression model:

$$
y(\underline{x})=\beta+Z(\underline{x}),
$$


where, the sampling point $\underline{x}$ has $k$-dimensions. $\beta$ is a global constant and $Z(\underline{x})$ is a stochastic process that is assumed to have mean zero. A covariance of $Z(\underline{x})$ between the $i$ th and $j$ th experiments is defined as

$$
\operatorname{Cov}\left[Z\left(\underline{x}_{i}\right), Z\left(\underline{x}_{j}\right)\right]=\delta^{2} R\left(\underline{x}_{i}, \underline{x}_{j}\right),
$$

where, $\delta^{2}$ is the variance of a stochastic process. The correlation function $R\left(\underline{x}_{i}, \underline{x}_{j}\right)$ between the sampling points has the form:

$$
R\left(\underline{x}_{i}, \underline{x}_{j}\right)=\exp \left(-\sum_{k=1}^{n} \theta_{k}\left|x_{i, k}-x_{j, k}\right|^{2}\right)
$$

where $\theta$ is a design parameter which should be determined for response surface design. Then, the response value for $n$-sampling points can be written in a vector form:

$$
\underline{y}=\beta+\underline{Z},
$$

where, $\underline{y}$ and $\underline{Z}$ corresponding to each sampling point $\underline{x}$ are

$$
\begin{aligned}
\underline{y} & =\left[y_{1}\left(\underline{x}_{1}\right), y_{2}\left(\underline{x}_{2}\right), y_{3}\left(\underline{x}_{3}\right), \cdots, y_{n}\left(\underline{x}_{n}\right)\right]^{T}, \\
\underline{Z} & =\left[Z_{1}\left(\underline{x}_{1}\right), Z_{2}\left(\underline{x}_{2}\right), Z_{3}\left(\underline{x}_{3}\right), \cdots, Z_{n}\left(\underline{x}_{n}\right)\right]^{T},
\end{aligned}
$$

respectively. Using determistic response $\underline{y}$ at given $n$-sampling points $\underline{x}$, the ordinary Kriging model considers a linear predictor $\hat{y}$ at untried $\underline{x}^{\prime}$ as follows

$$
\hat{y}\left(\underline{x}^{\prime}\right)=\underline{c}^{T} \underline{y} .
$$

Then, an optimized estimated response value ${ }^{7}$ at untried $\underline{x}^{\prime}$ can be written as

$$
\hat{y}\left(\underline{x}^{\prime}\right)=\underline{c}^{T} \underline{y}=\left[\begin{array}{ll}
\underline{\varsigma} & \underline{r}^{T}
\end{array}\right]\left[\begin{array}{cc}
0 & \underline{\underline{\varsigma}} \\
\underline{\underline{\varsigma}} & \underline{\underline{R}}
\end{array}\right]^{-1}\left[\begin{array}{l}
0 \\
\underline{y}
\end{array}\right] .
$$

After solving the matrix of Eq. (13), it can be rewritten as

$$
\hat{y}\left(\underline{x}^{\prime}\right)=\underline{\beta}+\underline{r}^{T} \underline{\underline{R}}^{-1}(\underline{y}-\underline{\underline{\varsigma}} \underline{\beta})
$$

where a correlation matrix $\underline{\underline{R}}$, correlation vector $\underline{r}$ for an untried $\underline{x}^{\prime}$, and generalized least square estimator vector $\underline{\beta}$ are defined as

$$
\underline{\underline{R}}=\left[\begin{array}{cccc}
R\left(\underline{x}_{1}, \underline{x}_{1}\right) & R\left(\underline{x}_{1}, \underline{x}_{2}\right) & \cdots & R\left(\underline{x}_{1}, \underline{x}_{n}\right) \\
R\left(\underline{x}_{2}, \underline{x}_{1}\right) & R\left(\underline{x}_{2}, \underline{x}_{2}\right) & \cdots & R\left(\underline{x}_{1}, \underline{x}_{n}\right) \\
\vdots & \vdots & \ddots & \vdots \\
R\left(\underline{x}_{n}, \underline{x}_{1}\right) & R\left(\underline{x}_{n}, \underline{x}_{2}\right) & \cdots & R\left(\underline{x}_{n}, \underline{x}_{n}\right)
\end{array}\right]
$$

American Institute of Aeronautics and Astronautics 


$$
\begin{gathered}
\underline{r}=\left[R\left(\underline{x}_{1}, \underline{x}^{\prime}\right), R\left(\underline{x}_{2}, \underline{x}^{\prime}\right), R\left(\underline{x}_{3}, \underline{x}^{\prime}\right), \cdots, R\left(\underline{x}_{n}, \underline{x}^{\prime}\right)\right]^{T}, \\
\underline{\beta}=\left(\underline{\underline{\varsigma}}^{T} \underline{\underline{R}}^{-1} \underline{\underline{\varsigma}}\right)^{-1} \underline{\underline{\varsigma}}^{T} \underline{\underline{R}}^{-1} \underline{y}
\end{gathered}
$$

respectively. $\underline{\underline{\varsigma}}$ is a matrix in which all components has unit value. Then, in estimating the response value $\hat{y}$ at untried $\underline{x}^{\prime}$, the only $\underline{\theta}=\left[\theta_{1}, \theta_{2}, \cdots, \theta_{k}\right]^{T}$ of Eq. (8) remains. This designed parameter $\underline{\theta}$ can be determined by maximizing the following likelihood function ${ }^{7}$

$$
L(\underline{\theta})=-\frac{1}{2}\left[n \ln \left(\delta^{2}\right)+\ln (\operatorname{det}(\underline{\underline{R}}))\right]
$$

where deviation $\delta^{2}$ is defined as

$$
\delta^{2}=\frac{1}{n}(\underline{y}-\underline{\underline{\varsigma}} \underline{\underline{\beta}})^{T} \underline{\underline{R}}^{-1}(\underline{y}-\underline{\underline{\varsigma}} \underline{\beta}) .
$$

Maximizing the likelihood function is an $n$-dimensional unconstrained non-linear optimization problem. In the present work, the hybrid genetic algorithm ${ }^{8,9}$ is adopted to solve this problem with the objective function as

$$
\begin{array}{ll}
\text { Minimize } & L(\underline{\theta})=\operatorname{det}(\underline{\underline{R}})^{\frac{1}{n}} \delta^{2} \\
\text { Subject to } & \theta_{j} \geq 0 \text { for } j=1, \cdots, k
\end{array}
$$

The sample points to design a predicted response surface of the state-to-state transition cross sections are evaluated by a QCT method. ${ }^{3,4} \mathrm{~A}$ total of 60 rovibrational states are randomly selected from the total of 348 rovibrational states of $\mathrm{H}_{2}$. Then 1,830 of the initial collision pairs of target and projectile particle are constructed to evaluate the state-to-state cross sections for the sample points. For this smaller number of initial collision pairs, the QCT calculations are performed for various relative translational energies from $0.1 \mathrm{eV}$ to $6 \mathrm{eV}$.

In the QCT calculations, a potential energy surface proposed by Schwenke ${ }^{10}$ is used. In determination of initial coordinates and conjugated momenta of $\mathrm{H}_{2}$, a fast Fourier transform method proposed by Eaker ${ }^{11}$ is adopted with some modifications. Final rotational and vibrational energy levels are quantized by the Wentzel, Kramers, and Brillouin approximation method. ${ }^{3}$ Quasi-bound states are treated by the effective potential method proposed by Kuntz, ${ }^{4}$ and the collisions are calculated in a classical manner. A total of 2,000 trajectories are calculated per impact parameter at the batch size of $0.1 \AA$. In ensuring the convergence of the cross sections, the calculations are repeated until all collisions became elastic. Reactive and nonreactive trajectories are treated separately in the calculations of the cross sections. The nonreactive trajectories are counted as described by Mandy et al., ${ }^{12}$ whereas the reactive trajectories are counted twice: the first as ortho-state with a weighting of 3/4 due to nuclear spin, and the second as para-state with a weighting of 1/4. Further details about the QCT calculations can be found in other literature. ${ }^{3,4}$

The predicted response surfaces are designed by the ordinary Kriging model from the sample cross sections for each relative translational energy. From the predicted response surfaces, the complete sets of the state-to-state transition cross sections are derived. These cross sections are converted to the transition rate coefficients by using Eqs. (2) to (5) for the temperature range from $1,000 \mathrm{~K}$ to $30,000 \mathrm{~K}$. In this design methodology, the required computing resources are significantly reduced in comparison to the exact calculating method. In the design methodology, 1,830 initial collision pairs are adopted, while in the exact calculating methodology, 60,726 initial collision pairs are needed. The computing resources are therefore reduced by about a factor of 33 .

In Fig. 1, the predicted response surfaces designed by the ordinary Kriging model and the sample points of the cross section data are shown. The contour surface is the predicted response surface and the red spheres are the sample points. The $\mathrm{x}$-axis and $\mathrm{y}$-axis are the rovibrational states of the target and projectile molecules, respectively. In all figures, it is shown that the predicted response surface of the cross sections seem to be designed well from those of the sample QCT calculations. 
In Fig. 2. the state-to-state transition cross sections derived from the predicted response surface are compared with the cross sections unused for the response surface design. In Figs. 2 (a) and (c), the comparisons are made along the rotational state, and in Figs. 2 (b) and (d), the comparisons are made along the vibrational state. All of the designed values are within the range of error bars of the exact QCT calculations.

In Fig. 3, the present designed cross sections are compared with cross sections calculated by Mandy and Pogrebnya. ${ }^{13}$ In the work of Mandy and Pogrebnya, the cross sections were calculated by a QCT method based on BKMP potential energy surface. ${ }^{14}$ This potential energy surface has an advantage to accurately describe a long range force over the Schwenke potential energy surface. ${ }^{10}$ However, the computational time cost of the BKMP potential energy surface is more expensive than that of the Schewenke potential energy surface. The present cross sections derived from the predicted response surface are similar to the cross sections calculated by Mandy and Pogrebnya. Especially, in Cases 1 to 3, the cross sections agree quite well with the QCT calculations. In the cross sections where the value is lower than a $10^{-3} a_{0}^{2}$, a discernible difference is shown. However, the values of these cross sections are small enough to ignore.

In Fig. 4, the present vibrational self-relaxation rates for para- $\mathrm{H}_{2}$ are compared with the transition rates of the theoretical calculations ${ }^{15,16}$ and experiments. ${ }^{17-19}$ The present vibrational self-relaxation rates are obtained by the method proposed by Zenevich et al. ${ }^{15}$ In the works of Zenevich et al., ${ }^{15}$ the rotational-vibrational energy transitions were calculated by a semi-classical method. In the theoretical calculations by Furudate et al., ${ }^{16}$ the DSMC-QCT method was adopted to calculate a vibrational self-relaxation rates. In the experiments by Audibert et al., ${ }^{17,18}$ the vibrational relaxation rates were measured by Raman two-photon excitation and Schlieren detection technique for para- and ortho $-\mathrm{H}_{2}$ in the temperature range $40-500 \mathrm{~K}$. In the experiments by Dove and Teitalbaum, ${ }^{19}$ the selfrelaxation rates were obtained from the vibrational relaxation time. In the comparisons of vibrational selfrelaxation rates for para- $\mathrm{H}_{2}$, it is shown that the present relaxation rates agree well with the relaxation rates by semi-classical calculations and experiments.

\section{Master equation study without chemical reactions}

A system of the master equations is constructed by using the state-to-state transition rate coefficients designed by the ordinary Kriging model. Then, state-resolved rovibrational kinetics are simulated by the system of the master equations. The master equation of the bound-bound transitions is defined as

$$
\frac{\partial n_{i}}{\partial t}=\sum_{j} \sum_{\ell} \sum_{m} g_{i j \ell m} K_{i j \rightarrow \ell m}\left(\frac{Q_{i} Q_{j}}{Q_{\ell} Q_{m}} n_{\ell} n_{m}-n_{i} n_{j}\right),
$$

where, $i$ and $j$ denote an initial rovibrational state of the target and projectile molecule, respectively. $\ell$ and $m$ are the final states, corresponding to $i$ and $j$ rovibrational states. By dividing both sides by the equilibrium number density of state $i, n_{i_{E_{q}}}$, and introducing the normalized population $\varphi_{i}=n_{i} / n_{i_{E_{q}}}$, Eq. (21) can be rewritten as

$$
\frac{\partial \varphi_{i}}{\partial t}=\sum_{j, \ell, m} n_{j_{E q}} g_{i j \ell m} K_{i j \rightarrow l m}\left[\varphi_{\ell} \varphi_{m}-\varphi_{i} \varphi_{j}\right]
$$

A parameter $g_{i j \ell m}$ is defined as

$$
g_{i j \ell m}=\left[1+\delta_{i j}\left(1-\delta_{\ell i}\right)\left(1-\delta_{m i}\right)\right]\left[1-\delta_{\ell i}\left(1-\delta_{i j}\right)\right]\left[1-\delta_{m i}\left(1-\delta_{i j}\right)\right],
$$

where $\delta$ is the Direc-delta function. This parameter can automatically prohibit the multiple counting of symmetric rovibrational states. The partition function of rovibrational state $i, Q_{i}$, has the form:

$$
Q_{i}=\varepsilon g_{e}(2 j+1) g_{s} \exp \left(-\frac{e_{i}}{k T}\right)
$$


Table 1. Initial heat bath conditions for bound-bound transitions

\begin{tabular}{cccc}
\hline \hline & \multicolumn{3}{c}{ Heat bath condition } \\
\cline { 2 - 4 } & $T_{r}=T_{v}(\mathrm{~K})$ & $T(\mathrm{~K})$ & Number density $\left(\mathrm{cm}^{-3}\right)$ \\
\hline Case 1 & $500 \mathrm{~K}$ & $1,000 \mathrm{~K}$ & $1.0 \times 10^{18}$ \\
Case 2 & $1,000 \mathrm{~K}$ & $2,000 \mathrm{~K}$ & $1.0 \times 10^{18}$ \\
Case 3 & $1,000 \mathrm{~K}$ & $3,000 \mathrm{~K}$ & $1.0 \times 10^{18}$ \\
Case 4 & $1,000 \mathrm{~K}$ & $4,000 \mathrm{~K}$ & $1.0 \times 10^{18}$ \\
Case 5 & $1,000 \mathrm{~K}$ & $5,000 \mathrm{~K}$ & $1.0 \times 10^{18}$ \\
Case 6 & $1,000 \mathrm{~K}$ & $6,000 \mathrm{~K}$ & $1.0 \times 10^{18}$ \\
Caes 7 & $1,000 \mathrm{~K}$ & $8,000 \mathrm{~K}$ & $1.0 \times 10^{18}$ \\
Case 8 & $1,000 \mathrm{~K}$ & $10,000 \mathrm{~K}$ & $1.0 \times 10^{18}$ \\
Case 9 & $1,000 \mathrm{~K}$ & $15,000 \mathrm{~K}$ & $1.0 \times 10^{18}$ \\
Case 10 & $1,000 \mathrm{~K}$ & $20,000 \mathrm{~K}$ & $1.0 \times 10^{18}$ \\
Case 11 & $1,000 \mathrm{~K}$ & $25,000 \mathrm{~K}$ & $1.0 \times 10^{18}$ \\
Case 12 & $1,000 \mathrm{~K}$ & $30,000 \mathrm{~K}$ & $1.0 \times 10^{18}$ \\
\hline \hline
\end{tabular}

where the nuclear spin degeneracy $g_{s}$ is 1 when $j$ has an even number and 3 when an odd number. The principle of detailed balance relation between forward and backward rates is invoked under the assumption of equilibrium, which leads to

$$
K_{i j \rightarrow \ell m} Q_{i} Q_{j}=K_{\ell m \rightarrow i j} Q_{\ell} Q_{m} .
$$

The normalized populations $\varphi$ for all 348 rovibrational states are calculated for a heating environment by the system of the master equations of Eq. (22). Initial heat bath conditions are tabulated in Table 1. Strong thermal nonequilibrium conditions are presented in this condition. In master equation calculations, it is assumed that rotational and vibrational excitations occur at an isothermal condition. An implicit time integration method accurate to third order along the diagonal and second order off-diagonal elements is used in integrating the resulting system of 348 ordinary differential equations.

In Fig. 5, the relaxations of rotational and vibrational temperatures and number densities by the master equation calculations are presented. In the present work, the energy-equivalent temperatures of the rotational and vibrational modes are adopted. These energy-equivalent temperatures can be determined by the averaged rotational and vibrational energies;

$$
\begin{gathered}
e_{r}=\frac{\sum_{j}\left[e_{i}-e_{v}(i)\right] n_{i_{E_{q}}} \rho_{i}}{\sum_{i} n_{i_{E q}} \rho_{i}}, \\
e_{v}=\frac{\sum_{j} e_{v}(i) n_{i_{E_{q}}} \rho_{i}}{\sum_{i} n_{i_{E_{q}}} \rho_{i}} .
\end{gathered}
$$

In figures (a) and (b), it is observed that the relaxation of the rotational mode is discernibly faster than the relaxation of the vibrational mode in Case 4. However, the rotational relaxation time becomes similar to that of vibration, when the equilibrium temperature increases. In figures (c) and (d), the normalized number density distributions of the rotational and vibrational modes by master equation calculations for Case 8 are compared with the Boltzmann distributions according to nonequilibrium temperature. For both the rotational and vibrational number density distributions, the nonequilibrium distributions of the master equation calculations are obviously different from the Boltzmann distributions in strong nonequilibrium conditions. After more convergence toward equilibrium, these differences become small. However, in the low rotational and vibrational states, where the most 
rovibrational number density exists, the nonequilibrium distributions agree well with the Boltzmann distributions in strong and weak nonequilibrium conditions.

In Fig. 6, the characteristic relaxation parameters of $p \tau_{r}$ and $p \tau_{v}$ calculated in the present work are compared with the experimental results ${ }^{19,20}$ and other theoretical calculations. ${ }^{16,21}$ The relaxation time of the rotational and vibrational modes are defined by the Landau-Teller form ${ }^{22}$ as

$$
\begin{aligned}
& \frac{\partial e_{r}}{\partial t}=\frac{e_{r}(T)-e_{r}}{\tau_{r}}, \\
& \frac{\partial e_{v}}{\partial t}=\frac{e_{v}(T)-e_{v}}{\tau_{v}},
\end{aligned}
$$

respectively. By equating Eqs. (28) and (29) with the results of the master equation calculations, the relaxation time can be determined with the $e$-folding collision number method. ${ }^{23}$ By multiplying the pressure, one obtains the characteristic rotational and vibrational relaxation parameters $p \tau_{r}$ and $p \tau_{v}$, respectively. The figure shows that the rotational relaxation parameters of the present work agree well with the experimental values by Lensch and Gronig. ${ }^{20}$ In the vibrational relaxation parameters, the present results also agree well with the experimental values by Dove and Teitelbaum ${ }^{19}$ at all measured temperatures. In comparisons of the present relaxation parameters with the previous theoretical calculations by Sharma ${ }^{21}$ and Furudate et al., ${ }^{16}$ the present results have discernible differences with the previous theoretical calculations. In comparisons with the results by Kim et al., ${ }^{2}$ the present relaxation parameters have similar values. However, for the temperatures of $1,000 \mathrm{~K}$ and $2,000 \mathrm{~K}$, the present relaxation parameters have more agreement in comparing with the experiments than with the parameters by Kim et al. In all experiments and theoretical calculations, it is observed that the rotational relaxation time is faster than that of vibration initially. However, these differences of relaxation time between the rotational and vibrational modes become small when the temperature increases.

\section{Master equation study with chemical reactions}

A system of the master equations with nonequilibrium chemical reactions can be constructed with the state-tostate transition rate coefficients in the following form;

$$
\begin{aligned}
\frac{\partial n_{i}}{\partial t}= & \sum_{j} \sum_{\ell} \sum_{m} g_{i j \ell m} K_{i j \rightarrow \ell m}\left(\frac{Q_{i} Q_{j}}{Q_{\ell} Q_{m}} n_{\ell} n_{m}-n_{i} n_{j}\right) \\
& +\sum_{j} \sum_{\ell} g_{i j \ell} K_{i j \rightarrow \ell c}\left[\frac{Q_{i}}{Q_{\ell}} \frac{Q_{t_{H_{2}}} Q_{j}}{Q_{t_{H}}^{2} Q_{H}^{2}} \exp \left(\frac{D_{j}-e_{j}}{k T}\right) n_{\ell} \frac{n_{H}^{2}}{4}-n_{i} n_{j}\right] \\
& +\sum_{j} \sum_{m} g_{i j \ell} K_{i j \rightarrow c m}\left[\frac{Q_{j}}{Q_{m}} \frac{Q_{t_{H_{2}}} Q_{i}}{Q_{t_{H}}^{2} Q_{H}^{2}} \exp \left(\frac{D_{i}-e_{i}}{k T}\right) n_{m} \frac{n_{H}^{2}}{4}-n_{i} n_{j}\right], \\
& +\sum_{j} g_{i j \ell} K_{i j \rightarrow c c}\left[\frac{Q_{t_{H_{2}} t_{H_{2}}}^{2} Q_{i} Q_{j}}{Q_{t_{H}}^{4} Q_{H}^{4}} \exp \left(\frac{D_{i}+D_{j}-e_{i}-e_{j}}{k T}\right) \frac{n_{H}^{4}}{16}-n_{i} n_{j}\right] \\
\frac{\partial n_{H}}{\partial t}= & \sum_{i} \sum_{j} \sum_{\ell} g_{i j \ell} K_{i j \rightarrow \ell c}\left[n_{i} n_{j}-\frac{Q_{i}}{Q_{\ell}} \frac{Q_{t_{H_{2}}} Q_{j}}{Q_{t_{H}}^{2} Q_{H}^{2}} \exp \left(\frac{D_{j}-e_{j}}{k T}\right) n_{\ell} \frac{n_{H}^{2}}{4}\right] \\
& +\sum_{i} \sum_{j} \sum_{m} g_{i j \ell} K_{i j \rightarrow c m}\left[n_{i} n_{j}-\frac{Q_{j}}{Q_{m}} \frac{Q_{t_{H_{2}}} Q_{i}}{Q_{t_{H}}^{2} Q_{H}^{2}} \exp \left(\frac{D_{i}-e_{i}}{k T}\right) n_{m} \frac{n_{H}^{2}}{4}\right], \\
& +\sum_{i} \sum_{j} g_{i j \ell} K_{i j \rightarrow c c}\left[n_{i} n_{j}-\frac{Q_{t_{H_{2}} t_{H_{2}}}^{2} Q_{i} Q_{j}}{Q_{t_{H}}^{4} Q_{H}^{4}} \exp \left(\frac{D_{i}+D_{j}-e_{i}-e_{j}}{k T}\right) \frac{n_{H}^{4}}{16}\right]
\end{aligned}
$$


where, the prohibiting parameters of $g_{i j \ell}, g_{i j m}$, and $g_{i j}$ are defined as

$$
\begin{gathered}
g_{i j \ell}=\left[1+\delta_{i j}\left(1-\delta_{\ell i}\right)\right]\left[1-\delta_{\ell i}\left(1-\delta_{i j}\right)\right], \\
g_{i j m}=\left[1+\delta_{i j}\left(1-\delta_{m i}\right)\right]\left[1-\delta_{m i}\left(1-\delta_{i j}\right)\right], \\
g_{i j}=\left[1+\delta_{i j}\right],
\end{gathered}
$$

respectively. The principle of detailed balancing relations for bound-free transitions are

$$
\begin{gathered}
K_{i j \rightarrow c c} \frac{Q_{i}}{Q_{\ell}} \frac{Q_{t_{H_{2}}} Q_{j}}{Q_{t_{H}}^{2} Q_{H}^{2}} \exp \left(\frac{D_{j}-e_{j}}{k T}\right)=K_{\ell c \rightarrow i, j} \\
K_{i j \rightarrow c m} \frac{Q_{j}}{Q_{m}} \frac{Q_{t_{H_{2}}} Q_{i}}{Q_{t_{H}}^{2} Q_{H}^{2}} \exp \left(\frac{D_{i}-e_{i}}{k T}\right)=K_{c m \rightarrow i, j} \\
K_{i j \rightarrow c c} \frac{Q_{t_{H_{2}} t_{H_{2}}}^{2} Q_{i} Q_{j}}{Q_{t_{H}}^{4} Q_{H}^{4}} \exp \left(\frac{D_{i}+D_{j}-e_{i}-e_{j}}{k T}\right)=K_{c c \rightarrow i j}
\end{gathered}
$$

By dividing both sides by the equilibrium number density, and introducing the normalized nonequilibrium population $\varphi_{i}=n_{i} / n_{i_{E q}}$ and $\varphi_{H}=n_{H} / n_{H_{E_{q}}}$, Eqs. (30) and (31) can be rewritten as

$$
\begin{aligned}
\frac{d \varphi_{i}}{d t}= & \sum_{j} \sum_{\ell} \sum_{m} g_{i j \ell m} n_{j_{E q}} K_{i j \rightarrow \ell m}\left(\varphi_{\ell} \varphi_{m}-\varphi_{i} \varphi_{j}\right) \\
& +\sum_{j} \sum_{\ell} g_{i j \ell} n_{j_{E q}} K_{i j \rightarrow \ell c}\left(\varphi_{\ell} \frac{\varphi_{H}^{2}}{4}-\varphi_{i} \varphi_{j}\right)+\sum_{j} \sum_{m} g_{i j m} n_{j_{E q}} K_{i j \rightarrow c m}\left(\varphi_{m} \frac{\varphi_{H}^{2}}{4}-\varphi_{i} \varphi_{j}\right), \\
& +\sum_{j} g_{i j} K_{i j \rightarrow c c}\left(\frac{\varphi_{H}^{4}}{16}-\varphi_{i} \varphi_{j}\right) \\
\frac{d \varphi_{H}}{d t}= & \sum_{i} \sum_{j} \sum_{\ell} \frac{2 n_{i_{E q}} n_{j_{E q}}}{n_{H_{E q}}} K_{i j \rightarrow \ell c}\left(\varphi_{i} \varphi_{j}-\varphi_{\ell} \frac{\varphi_{H}^{2}}{4}\right)+\sum_{i} \sum_{j} \sum_{m} \frac{2 n_{i_{E q}} n_{j_{E q}}}{n_{H_{E q}}} K_{i j \rightarrow c m}\left(\varphi_{i} \varphi_{j}-\varphi_{m} \frac{\varphi_{H}^{2}}{4}\right), \\
+ & \sum_{i} \sum_{j} \frac{4 n_{i_{E q}} n_{j_{E q}}}{n_{H_{E_{q}}}} K_{i j \rightarrow c c}\left(\varphi_{i} \varphi_{j}-\frac{\varphi_{H}^{4}}{16}\right)
\end{aligned}
$$

respectively.

All normalized populations $\varphi$ of Eqs. (35) and (36) are calculated for heating and cooling environments in a heat bath. Initial conditions of these heating and cooling environments are tabulated in Table 2 . The initial number densities of $\mathrm{H}_{2}$ and $\mathrm{H}$ are set to a constant as $1.0 \times 10^{18} \mathrm{~cm}^{-3}$ and zero, respectively. Input conditions of number densities of each rovibrational state and each species are determined by equilibrium chemical reactions before putting into the system of master equations. In the computational method, an implicit time integration method accurate to third order along the diagonal and second order off-diagonal elements is adopted in integrating the 349 ordinary differential equations of Eqs. (35) and (36).

In Fig. 7, the relaxations of the rotational and vibrational temperatures and number densities by master equation calculations are presented for the heating cases. In figures (a) and (b), the nonequilibrium chemical reactions in QSS are observed above 6,000K cases. During this QSS period, the rotational and vibrational temperatures are at almost constant values, and the number density of $\mathrm{H}$ is rapidly increased. In figures (c) and (d), the rotational and vibrational number density distributions at seven points in Case 8 are shown. In both the rotational and vibrational cases, the number density distributions seem to be frozen in QSS. This is because QSS occurs when the number density rate of change $n_{i}$ on the left-hand side in Eq. (30) is much smaller than both the sum of all incoming rates and sum of all outgoing rates on the right-hand side.

American Institute of Aeronautics and Astronautics 
Table 2. Initial heat bath conditions for heating and cooling environments

\begin{tabular}{ccccc}
\hline \hline & \multicolumn{2}{c}{ Heating condition } & \multicolumn{2}{c}{ Cooling condtion } \\
\cline { 2 - 5 } & $T_{r}=T_{v}(K)$ & $T(K)$ & $T_{r}=T_{v}(K)$ & $T(K)$ \\
\hline Case 1 & $500 \mathrm{~K}$ & $1,000 \mathrm{~K}$ & $10,000 \mathrm{~K}$ & $2,000 \mathrm{~K}$ \\
Case 2 & $1,000 \mathrm{~K}$ & $2,000 \mathrm{~K}$ & $10,000 \mathrm{~K}$ & $3,000 \mathrm{~K}$ \\
Case 3 & $1,000 \mathrm{~K}$ & $3,000 \mathrm{~K}$ & $10,000 \mathrm{~K}$ & $4,000 \mathrm{~K}$ \\
Case 4 & $1,000 \mathrm{~K}$ & $4,000 \mathrm{~K}$ & $10,000 \mathrm{~K}$ & $5,000 \mathrm{~K}$ \\
Case 5 & $1,000 \mathrm{~K}$ & $5,000 \mathrm{~K}$ & $10,000 \mathrm{~K}$ & $6,000 \mathrm{~K}$ \\
Case 6 & $1,000 \mathrm{~K}$ & $6,000 \mathrm{~K}$ & $10,000 \mathrm{~K}$ & $8,000 \mathrm{~K}$ \\
Case 7 & $1,000 \mathrm{~K}$ & $8,000 \mathrm{~K}$ & & \\
Case 8 & $1,000 \mathrm{~K}$ & $10,000 \mathrm{~K}$ & & \\
Case 9 & $1,000 \mathrm{~K}$ & $15,000 \mathrm{~K}$ & & \\
Case 10 & $1,000 \mathrm{~K}$ & $20,000 \mathrm{~K}$ & & \\
Case 11 & $1,000 \mathrm{~K}$ & $25,000 \mathrm{~K}$ & & \\
Case 12 & $1,000 \mathrm{~K}$ & $30,000 \mathrm{~K}$ & & \\
\hline \hline
\end{tabular}

In Fig. 8, the relaxations of the rotational and vibrational temperatures and number densities by master equation calculations are presented for the cooling cases. In figures (a) and (b), the rotational and vibrational temperatures locally increase. This is because, in this local period, the number densities of high-rotational and vibrational energy levels are increased due to recombination, and this recombination occurs at a much faster rate than the rotational and vibrational relaxations. These phenomena are also accounted for in figures (c) and (d). In figures (c) and (d), the vibrational relaxation rates and the normalized number density distributions of the vibrational mode for Case 2 are presented, respectively. The vibrational relaxation rates of high-vibrational energy levels are larger than those of the low-vibrational energy levels. However, slow relaxations of vibrational number density are observed on highvibrational energy levels than on low-vibrational energy levels.

In Fig. 9, the dissociation and recombination reaction rates of the present work are compared with experiments ${ }^{24-}$ ${ }^{29}$ and theoretical calculations. ${ }^{2,10,16}$ The present dissociation reaction rates are obtained in QSS, and the recombination rates are obtained where the recombination occurs rapidly. In the comparisons of dissociation reaction rates, the present rates agree well with the recommended experimental values by Cohen and Westburg ${ }^{24}$ and theoretical calculations by Kim et $\mathrm{al}^{2}{ }^{2}$ However, the present reaction rates have discernible differences with the oneway rates ${ }^{30}$ defined as

$$
K_{f_{\text {one-way }}}=\sum_{i, j, \ell} \frac{Q_{i} Q_{j}}{Q_{m}^{2}} K_{i j \rightarrow \ell c}+\sum_{i, j, m} \frac{Q_{i} Q_{j}}{Q_{m}^{2}} K_{i j \rightarrow c m}+\sum_{i, j} \frac{Q_{i} Q_{j}}{Q_{m}^{2}} K_{i j \rightarrow c c} .
$$

This is because the present reactions rates are determined in a nonequilibrium process and the one-way rates are determined in a equilibrium process. In the recombination reaction rates, the experimental results ${ }^{25-29}$ are shown to be scattered over a logarithmic range between 14.7 and 15.4 at temperatures from 3000 to $5000 \mathrm{~K}$. The theoretical recombination rate coefficients of the present work fall within this boundary. However, the theoretical calculations of Schwenke ${ }^{10}$ and Furudate et al. ${ }^{16}$ either overpredict or underpredict the experimental data.

In Fig. 10, the averaged rotational and vibrational energy losses due to dissociation are presented. Using the detailed balancing relations of Eqs. (35) to (37) and the rate of change of atomic number density in Eq. (31), the averaged energy loss of the rotational and vibrational modes can be expressed as 


$$
\begin{aligned}
& \sum_{i, j, \ell} n_{i_{E q}} n_{j_{E_{q}}} e_{X}(j) K_{i j \rightarrow \ell c}\left[\varphi_{i} \varphi_{j}-\varphi_{\ell} \frac{\varphi_{H}^{2}}{4}\right]+\sum_{i, j, m} n_{i_{E_{q}}} n_{j_{E q}} e_{X}(i) K_{i j \rightarrow c m}\left[\varphi_{i} \varphi_{j}-\varphi_{m} \frac{\varphi_{H}^{2}}{4}\right] \\
& e_{X}=\frac{+\sum_{i, j} n_{i_{E_{q}}} n_{j_{E_{q}}}\left[e_{X}(i)+e_{X}(j)\right] K_{i j \rightarrow c c}\left[\varphi_{i} \varphi_{j}-\frac{\varphi_{H}^{4}}{16}\right]}{\sum_{i, j, \ell} n_{i_{E_{q}}} n_{j_{E_{q}}} K_{i j \rightarrow \ell c}\left[\varphi_{i} \varphi_{j}-\varphi_{\ell} \frac{\varphi_{H}^{2}}{4}\right]+\sum_{i, j, m} n_{i_{E_{q}}} n_{j_{E_{q}}} K_{i j \rightarrow c m}\left[\varphi_{i} \varphi_{j}-\varphi_{m} \frac{\varphi_{H}^{2}}{4}\right]}, \\
&+\sum_{i, j} n_{i_{E_{q}}} n_{\sum_{E_{q}}} K_{i j \rightarrow c c}\left[\varphi_{i} \varphi_{j}-\frac{\varphi_{H}^{4}}{16}\right]
\end{aligned}
$$

where, $X$ represents $r$ for averaged rotational energy loss, and $v$ for averaged vibrational energy loss. The averaged rovibrational energy loss is the summation of the energy losses of both modes. In the present work, it is observed that when the temperature increases, the rotational energy loss also increases, however the vibrational energy and rovibrational energy losses decrease. This is because, at low temperatures, most of the dissociation occurs at highvibrational and low-rotational energy levels. When the temperature increases, the averaged dissociated energy levels move to lower vibrational and higher rotational energy levels. In the present work, the ratio of averaged energy loss divided by dissociation energy converge to $0.35,0.50,0.85$ for rotation, vibration, and coupled rovibration modes, respectively. In comparisons with the results by Kim et al. ${ }^{2}$ and Furudate et al., ${ }^{16}$ discernible differences are observed. The converged patterns of the present averaged rovibrational energy loss is totally different from those by Kim et al.

\section{Post-normal shock and nozzle expanding flows}

A system of master equations are coupled with one-dimensional flow equations to analyze the relaxations of $\mathrm{H}_{2}$ in post-normal shock and nozzle expanding flows. The master equations for $\mathrm{H}, \mathrm{He}$, and $\mathrm{H}_{2}$ mixtures are considered in coupling with the one-dimensional flow equations. In the present work, radiations of $\mathrm{H}, \mathrm{He}$, and $\mathrm{H}_{2}$ are ignored to simplify the coupled equations. Mass, momentum and energy conservation equations with one-dimensional, steadystate, and isentropic assumptions are derived as

$$
\begin{gathered}
\frac{1}{\rho} \frac{d \rho}{d x}=-\left[\frac{1}{u^{2}} \frac{d}{d x}\left(\frac{u^{2}}{2}\right)+\frac{1}{A} \frac{d A}{d x}\right], \\
\frac{d}{d x}\left(\frac{u^{2}}{2}\right)=-\frac{1}{\rho} \frac{d p}{d x}, \\
u \frac{d h}{d x}=0
\end{gathered}
$$

where, pressure and enthalpy are defined as

$$
\begin{gathered}
p=\sum_{i s p}^{H, H e, H_{2}} \rho N_{A} \gamma_{i s p} k T, \\
h=\sum_{i s p}^{H, H e, H_{2}} h_{i s p}^{f} \gamma_{i s p}+\frac{5}{2} N_{A} k T \sum_{i s p}^{H, H e, H_{2}} \gamma_{i s p}+\sum_{i s p}^{H_{2}} N_{A}\left(e_{r} \gamma_{i s p}+e_{v} \gamma_{i s p}\right)+\frac{u^{2}}{2},
\end{gathered}
$$

respectively. A system of master equations are coupled with the one-dimensional flow equations as species and rovibrational concentration equations; 
Table 3. Initial conditions of the nozzle expanding and post-normal shock flows

\begin{tabular}{lcccc}
\hline \hline Post-normal shock, Case 1 & $u_{\infty}=20.0 \mathrm{~km} / \mathrm{sec}$ & $p_{\infty}=0.1$ Torr & - & $T_{\infty}=500 \mathrm{~K}$ \\
Post-normal shock, Case 2 & $u_{\infty}=30.0 \mathrm{~km} / \mathrm{sec}$ & $p_{\infty}=0.1$ Torr & - & $T_{\infty}=500 \mathrm{~K}$ \\
Nozzle expanding, Case 1 & - & $p_{s}=10.0 \mathrm{~atm}$ & $h_{s}=100.0 \mathrm{MJ} / \mathrm{kg}$ & - \\
Nozzle expanding, Case 2 & - & $p_{s}=10.0 \mathrm{~atm}$ & $h_{s}=200.0 \mathrm{MJ} / \mathrm{kg}$ & - \\
\hline \hline
\end{tabular}

$$
\begin{aligned}
& u \frac{d \gamma_{i}}{d x}=\sum_{j, \ell, m} g_{i, j, \ell, m} \rho N_{A} K_{i j \rightarrow \ell m}^{H_{2}+H_{2}}\left[\frac{Q_{i} Q_{j}}{Q_{\ell} Q_{m}} \gamma_{\ell} \gamma_{m}-\gamma_{i} \gamma_{j}\right] \\
& +\sum_{j, \ell} g_{i, j, \ell} \rho N_{A} K_{i j \rightarrow \ell c}^{H_{2}+H_{2}}\left[\frac{Q_{i}}{Q_{\ell}} \frac{Q_{t_{\ell}} Q_{j}}{Q_{t_{H}}^{2} Q_{H}^{2}} \exp \left(\frac{D_{j}-e_{j}}{k T}\right) \rho N_{A} \gamma_{\ell} \frac{\gamma_{H}^{2}}{4}-\gamma_{i} \gamma_{j}\right] \\
& +\sum_{j, m} g_{i, j, m} \rho N_{A} K_{i j \rightarrow c m}^{H_{2}+H_{2}}\left[\frac{Q_{j}}{Q_{m}} \frac{Q_{t_{H}} Q_{i}}{Q_{t_{H}^{2}}^{2} Q_{H}^{2}} \exp \left(\frac{D_{i}-e_{i}}{k T}\right) \rho N_{A} \gamma_{m} \frac{\gamma_{H}^{2}}{4}-\gamma_{i} \gamma_{j}\right] \\
& +\sum_{j} g_{i, j} \rho N_{A} K_{i j \rightarrow c c}^{H_{2}+H_{2}}\left[\frac{Q_{t_{H_{2}}}^{2} Q_{i} Q_{j}}{Q_{t_{H}}^{4} Q_{H}^{4}} \exp \left(\frac{D_{i}+D_{j}-e_{i}-e_{j}}{k T}\right) \rho^{2} N_{A}^{2} \frac{\gamma_{H}^{4}}{16}-\gamma_{i} \gamma_{j}\right], \\
& +\sum_{M}^{H, H e} \rho N_{A} K_{i \rightarrow \ell}^{M+H_{2}}\left[\frac{Q_{i}}{Q_{\ell}} \gamma_{\ell} \gamma_{M}-\gamma_{i} \gamma_{M}\right] \\
& +\sum_{M}^{H, H e} \rho N_{A} K_{i \rightarrow c}^{M+H_{2}}\left[\frac{Q_{t_{2}} Q_{i}}{Q_{t_{H}}^{2} Q_{H}^{2}} \exp \left(\frac{D_{i}-e_{i}}{k T}\right) \rho N_{A} \gamma_{M} \frac{\gamma_{H}^{2}}{4}-\gamma_{i} \gamma_{M}\right] \\
& u \frac{d \gamma_{H}}{d x}=\sum_{i, j, \ell} 2 \rho N_{A} K_{i j \rightarrow \ell c}^{H_{2}+H_{2}}\left[\gamma_{i} \gamma_{j}-\frac{Q_{i}}{Q_{\ell}} \frac{Q_{t_{H_{2}}} Q_{j}}{Q_{t_{H}}^{2} Q_{H}^{2}} \exp \left(\frac{D_{j}-e_{j}}{k T}\right) \rho N_{A} \gamma_{\ell} \frac{\gamma_{H}^{2}}{4}\right] \\
& +\sum_{i, j, m} 2 \rho N_{A} K_{i j \rightarrow c m}^{H_{2}+H_{2}}\left[\gamma_{i} \gamma_{j}-\frac{Q_{j}}{Q_{m}} \frac{Q_{t_{H_{2}}} Q_{i}}{Q_{t_{H}}^{2} Q_{H}^{2}} \exp \left(\frac{D_{i}-e_{i}}{k T}\right) \rho N_{A} \gamma_{m} \frac{\gamma_{H}^{2}}{4}\right] \\
& +\sum_{i j} 2 \rho N_{A} K_{i j \rightarrow c c}^{H_{2}+H_{2}}\left[\gamma_{i} \gamma_{j}-\frac{Q_{t_{H_{2}}}^{2} Q_{i} Q_{j}}{Q_{t_{H}}^{4} Q_{H}^{4}} \exp \left(\frac{D_{i}+D_{j}-e_{i}-e_{j}}{k T}\right) \rho^{2} N_{A}^{2} \frac{\gamma_{H}^{4}}{16}\right] \text {, } \\
& +\sum_{M}^{H, H e} \sum_{i} 2 \rho N_{A} K_{i \rightarrow c}^{M+H_{2}}\left[\gamma_{i} \gamma_{M}-\frac{Q_{H_{2}} Q_{i}}{Q_{t_{H}}^{2} Q_{H}^{2}} \exp \left(\frac{D_{i}-e_{i}}{k T}\right) \rho N_{A} \gamma_{M} \frac{\gamma_{H}^{2}}{4}\right] \\
& u \frac{d \gamma_{H e}}{d x}=0,
\end{aligned}
$$

In describing the rate of change of the rovibrational and species concentrations by $\mathrm{H}+\mathrm{H}_{2}$ and $\mathrm{He}+\mathrm{H}_{2}$ collisions, we use the state-to-state transition rate coefficients calculated by Kim et al. ${ }^{1}$

In the present work, one-dimensional flow codes are developed by using Eqs. (39) to (46). In the nozzle flow code, it is constructed of a settling chamber, subsonic nozzle, and supersonic nozzle parts. In the settling chamber part, calculations of equilibrium chemical reactions are performed by using the JANNAF thermochemical data base ${ }^{31}$ and the rigorous method of the Saha equation. ${ }^{30}$ In the subsonic nozzle part, equilibrium assumptions are adopted in onedimensional flow calculation. In the supersonic nozzle part, nonequilibrium thermochemical calculations are performed by coupling with one-dimensional flow and a system of master equations. In the post-normal shock flow code, it is constructed of two-parts; in the first part, jump conditions of the flow properties are determined by normal-shock relation equations. In the second part, the thermochemical nonequilibrium flows are calculated by the coupled equations in the post-normal shock region.

In Table 3, initial conditions of post-normal shock and nozzle expanding flows are tabulated. By these conditions, the mixture gases of settling chamber and free stream flow consist of $80 \% \mathrm{H}_{2}-20 \% \mathrm{He}$ by mole-fraction. This ratio is 
similar to those of the atmospheres of outer planets. In the post-normal shock flow conditions, a free steam velocity of 20 and $30 \mathrm{~km} / \mathrm{sec}$ are considered. This velocity is similar to the re-entry velocity into Neptune. In the nozzle expanding flows, a nozzle geometry of $10 \mathrm{~cm}$ length with $20 \mathrm{deg}$ of convergence-divergence nozzle types is adopted. The area ratio of nozzle throat and exit, $A_{e x i t} / A_{t h}$, is about 155.3. For all flow calculations, an implicit integration method accurate to third order along the diagonal and second order off-diagonal elements is adopted in integrating the mass, momentum, energy, and rovibrational and species concentration equations.

In Fig. 11, the results of temperatures and species mole-fractions of post-normal shock flows are presented. In the $20 \mathrm{~km} / \mathrm{sec}$ case, the relaxation of the rotational mode is slightly faster than that of vibration. These relaxations of the rotational and vibrational modes mostly occur within a distance of about $2 \mathrm{~cm}$ after the normal shock. In this region, the relaxations of the rotational and vibrational modes are dominantly affected by $\mathrm{H}_{2}+\mathrm{H}_{2}$ collisions. In $30 \mathrm{~km} / \mathrm{sec}$ case, the relaxation of the rotational mode is almost similar to the relaxation of the vibrational mode, and these relaxations are affected by all species collisions of $\mathrm{H}+\mathrm{H}_{2}, \mathrm{He}+\mathrm{H}_{2}$, and $\mathrm{H}_{2}+\mathrm{H}_{2}$.

In Fig. 12, the relaxations of temperatures and species mole-fractions are shown. In figures (a) and (b), temperature cooling occurs rapidly within a length of about $4 \mathrm{~cm}$ from the nozzle throat. In the length from $4 \mathrm{~cm}$ to $10 \mathrm{~cm}$, the relaxations of both rotational and vibrational modes seem to freeze. In this freezing region, the recombination process is obviously shown. These freezing phenomena are accounted for in figures (c) to (f). In figures (c) and (d), the normalized number density distributions and concentrations of the vibrational mode for $100 \mathrm{MJ} / \mathrm{kg}$ case are presented. In figures (e) and (f), those of the rotational mode are presented. When the flow is cooled in a supersonic nozzle, the recombination reactions occurs rapidly. The recombined molecules are present at high-rotational and vibrational energy levels. However, these molecules are slowly relaxed to lower energy levels. This is because the rotational and vibrational transition rates in low-energy levels are smaller than those of the highenergy levels in the temperature ranges where the recombination process occurs actively.

\section{Conclusion and future work}

The complete set of state-to-state transition rate coefficients for both target and projectile molecules are derived from the predicted response surface designed by the ordinary Kriging model. Sample cross sections used for designing the response surface are calculated by a quasi-classical trajectory method with randomly selected 1,830 initial collision pairs of rovibrational states. By adopting this design method, a computational cost is drastically reduced by a factor of 33 compare to the exact calculation. A system of master equations is constructed for boundbound and bound-free transitions with these transition rate coefficients, and the rovibrational number densities are numerically evaluated by solving a system of master equations. In comparisons with experiments, the present results of master equation calculations agree closer with the existing experimental data for rotational and vibrational relaxations and reaction rate coefficients for dissociation and recombination. From the results of master equation studies without chemical reactions, it is observed that the rotational relaxation time is faster than that of vibration initially. However, these differences of relaxation time between the rotation and vibration modes become small when the temperature increases. From the results of master equation studies with chemical reactions, it is observed that most dissociation of $\mathrm{H}_{2}$ occurred in a quasi-steady state. A local increase of the rotational and vibrational temperatures by recombined molecules is also observed in cooling processes. A system of master equations are coupled with one-dimensional flow equations to analyze the relaxations of $\mathrm{H}_{2}$ in post-normal shock and nozzle expanding flows. In post-normal shock flows, the relaxation of the rotational mode is slightly faster or similar to the relaxation of the vibrational mode. In nozzle expanding flows, the relaxations of both the rotational and vibrational modes seem to freeze in the supersonic nozzle. This is because the rotational and vibrational transition rates in low-energy levels are smaller than those of the high-energy levels where most recombined molecules exist.

In the present work, the master equation studies and flow analyses of post-normal shock and nozzle expanding flows are performed for $\mathrm{H}_{2}$ and $\mathrm{He}$ systems. In the future, these studies will be extended to analyze the same nonequilibrium processes for air species

\section{Acknowledgments}

The authors would like to thanks Hyoungil Kwon at KAIST in Republic of Korea for helpful comments and suggestions about the ordinary Kriging model. The authors gratefully acknowledge funding for this work through Air Force Office of Scientific Research Grant FA-9550-11-1-0309. 


\section{References}

${ }^{1}$ Kim, J. G., Kwon, O. J., and Park, C., "Master Equation Study and Non-equilibrium Chemical Reactions for $\mathrm{H}+\mathrm{H}_{2}$ and $\mathrm{He}+\mathrm{H}_{2}$," Journal of Thermophysics and Heat Transfer, Vol. 23, No. 3, 2009, pp. 443-453.

${ }^{2}$ Kim, J. G., Kwon, O. J., and Park, C., "Master Equation Study and Non-equilibrium Chemical Reactions for Hydrogen Molecule," Journal of Thermophysics and Heat Transfer, Vol. 24, No. 2, 2010, pp. 281-290.

${ }^{3}$ Bernstein, R. B., Atom-Molecule Collision Theory-A Guide for the Experimentalist, Plenum Press, New York, 1979.

${ }^{4}$ Miller, W. H., Dynamics of Molecular Collisions, Plenum Press, New York, 1976.

${ }^{5}$ Krige, D. G, "A Statistical, Approach To Some Basic Mine Valuation Problems on the Witwatersrand," Journal of the Chemical, Metallurgical and Mining Society of South Africa, Vol. 52, No. 6, 1951, pp. 119-139.

${ }^{6}$ Sacks, J., Welch, W. J., Mitchell, T. J., and Wynn, H. P., "Design and Analysis of Computer Experiments," Statistical Science, Vol. 4, No. 4, 1989, pp. 409-435.

${ }^{7}$ Kwon, H., "Kriging Model Based Aerodynamic Optimization for Wind Turbine Rotor Blade," Master's thesis, Aerospace Dept., KAIST, Daejeon, South Korea, 2011.

${ }^{8}$ Pham D. T. and Jin, G., "Genetic Algorithm using Gradient-like Reproduction Operator," Electronics Letters, Vol. 31, No. 18, 1995, pp. 1558-1559.

${ }^{9}$ Pham D. T. and Jin, G., "Hybrid Genetic Algorithm," Proceedings of 3rd World Congress on Expert Systems, Seoul, Korea, Vol. 2, 1996, pp. 748-757.

${ }^{10}$ Schwenke, D.W., "Calculations of Rate Constants for the Three-Body Recombination of $\mathrm{H}_{2}$ in the Presence of $\mathrm{H}_{2}$," Journal of Chemical Physics, Vol. 89, No. 4, 1988, pp. 2076-2091.

${ }^{11}$ Eaker, C. W., "A Fast Fourier Transform Method for Quasiclassical Selection of Initial Coordinates and Momenta for Rotating Diatoms," Journal of Chemical Physics, Vol. 90, No. 1, 1989, pp. 105-111.

${ }^{12}$ Mandy, M. E., Martin, P. G., and Keogh, W. J., "Why Quasiclassical Cross Sections Can Be Rotationally and Vibrationally Hot," Journal of Chemical Physics, Vol. 100, No. 4, 1994, pp. 2671-2676.

${ }^{13}$ Mandy, M. E., Pogrebnya, S. K., "Inelastic Collisions of Molecular Hydrogen: A Comparison of Results from Quantum and Classical Mechanics," Journal of Chemical Physics, Vol. 120, No. 12, 2004, pp. 5585-5591.

${ }^{14}$ Boothroyd, A. I., Keogh, W. J., Martin, P. G., and Peterson, M. R., "An Accurate Analytic $\mathrm{H}_{4}$ Potential Energy Surface," Journal of Chemical Physics, Vol. 116, No. 2, 2002, pp. 666-689.

${ }^{15}$ Zenevich, V. A., Billing, G. D., and Jolicard, G., "Vibrational-Rotational Energy Transfer in $\mathrm{H}_{2}-\mathrm{H}_{2}$ collisions II. The Relative Roles of the Initial Rotational Excitation, of Both Diatoms," Chemical Physics Letters, Vol. 312, No. 5-6, 1999, pp. 530535.

${ }^{16}$ Furudate, M., Fujita, K., and Abe, T., "Coupled Rotational-Vibrational Relaxation of Molecular Hydrogen at High Temperatures," Journal of Thermophysics and Heat Transfer, Vol. 20, No. 3, 2006, pp. 457-464.

${ }^{17}$ Audibert, M. M., Joffrin, C., and Ducuing, J., "Vibrational Relaxation of H2 in the Range 500-40K," Chemical Physics Letters, Vol. 25, No. 2, 1974, pp. 158-163.

${ }^{18}$ Audibert, M. M., Vilaseca, R., Lukasik, J., Ducuing, J., "Vibrational Relaxation of Ortho and Para- $\mathrm{H}_{2}$ in the Range 40050K," Chemical Physics Letters, Vol. 31, No. 2, 1975, pp. 232-236.

${ }^{19}$ Dove, J. E., and Teitelbaum, H., "The Vibrational Relaxation of $\mathrm{H}_{2}$, I. Experimental Measurements of the Rate of Relaxation by $\mathrm{H}_{2}, \mathrm{He}, \mathrm{Ne}$, Ar, and Kr," Chemical Physics, Vol. 6, No. 3, 1974, pp. 431-444.

${ }^{20}$ Lensch, G., and Gronig, H., "Experimental Determination of Rotational Relaxation in Molecular Hydrogen and Deuterium," Proceedings of the 11th International Symposium of Shock Tubes and Waves, Edited by B. Ahlborn, A. Hertzberg, and D. Russell, Univ. of Washington Press, Seattle, WA, 1977, pp. 132-139.

${ }^{21}$ Sharma, S. P., "Rotational Relaxation of Molecular Hydrogen at Moderate Temperatures," Journal of Thermophysics and Heat Transfer, Vol. 8, No. 1, 1994, pp. 35-39.

${ }^{22}$ Landau, L. and Teller, E., "Theory of Sound Dispersion,” Physics Sowjetunion, Vol. 10, 1936, pp. 34-43.

${ }^{23}$ Park, C., "Rotational Relaxation of $\mathrm{N}_{2}$ Behind a Strong Shock Wave," Journal of Thermophysics and Heat Transfer, Vol. 18, No. 4, 2004, pp. 527-533.

${ }^{24}$ Cohen, N. and Westberg, K. R., "Chemical Kinetic Data Sheets for High-Temperature Chemical Reactions," Journal of Physical Chemistry Reference Data, Vol. 12, No. 3, 1983, pp. 531-1267.

${ }^{25}$ Hurle, I. R., "Measurements of Hydrogen-Atom Recombination Rates Behind Shock Waves," 11th Symposium (International) on Combustion, Combustion Inst., Pittsburgh, PA, 1967, pp. 827-836.

${ }^{26}$ Jacobs, T. A., Giedt, R. R., and Cohen, N., "Kinetics of Hydrogen Halides in Shock Waves. II. A New Measurement of Hydrogen Dissociation Rate," Journal of Chemical Physics, Vol. 47, No. 1, 1967, pp. 54-57.

${ }^{27}$ Patch, R. W., "Shock-Tube Measurement of Dissociation Rates of Hydrogen," Journal of Chemical Physics, Vol. 36, No. 7, 1962, pp. 1919-1924.

${ }^{28}$ Rink, J. P., "Shock Tube Determination of Dissociation Rates of Hydrogen," Journal of Chemical Physics, Vol. 36, No. 1, 1962, pp. 262-265.

${ }^{29}$ Sutton, E. A., "Measurement of the Dissociation Rates of Hydrogen and Deuterium," Journal of Chemical Physics, Vol. 36, No. 11, 1962, pp. 2923-2931.

${ }^{30}$ Park, C., Nonequilibrium Hypersonic Aerothermodynamics, Wiley, New York, 1990.

${ }^{31}$ Perini, L., L., "Curve Fits of JANAF Thermochemical Data," Johns Hopkins Univ. Applied Physics Lab., Rept. ANSP-M-5, Silver Spring, MD, Sept. 1972. 

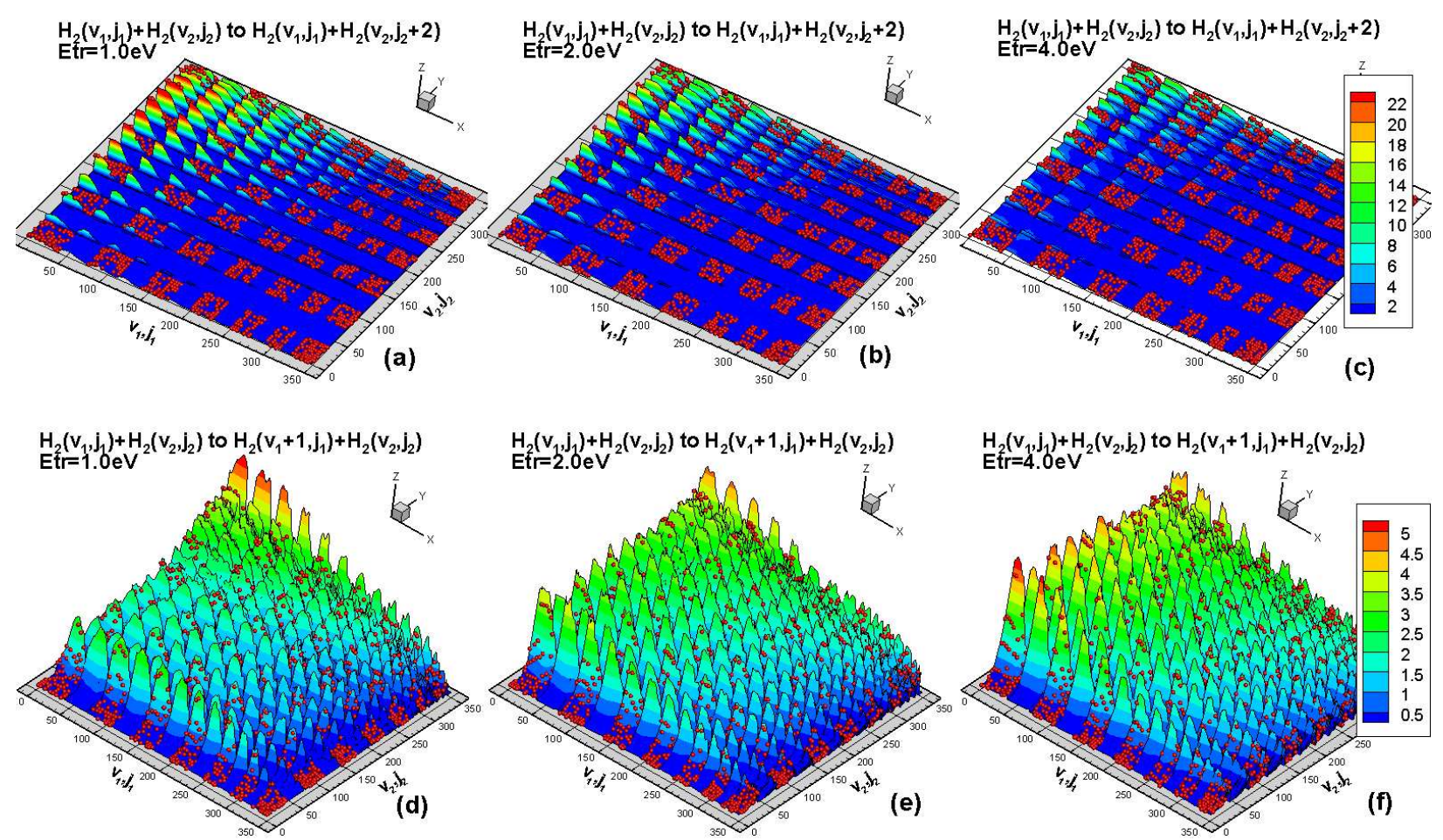

Figure 1. Response surfaces and sample cross sections for the transitions of $\mathbf{H}_{2}\left(\mathbf{v}_{1}, \mathbf{j}_{1}\right)+\mathbf{H}_{2}\left(\mathbf{v}_{2}, \mathbf{j}_{2}\right) \rightarrow \mathbf{H}_{2}\left(\mathbf{v}_{1}\right.$, $\left.\mathbf{j}_{1}\right)+\mathrm{H}_{2}\left(\mathbf{v}_{2}, \mathbf{j}_{2}+2\right)$ and $\mathbf{H}_{2}\left(\mathbf{v}_{1}, \mathbf{j}_{1}\right)+\mathbf{H}_{2}\left(\mathbf{v}_{2}, \mathbf{j}_{2}\right) \rightarrow \mathbf{H}_{2}\left(\mathbf{v}_{1}+1, \mathbf{j}_{1}\right)+\mathbf{H}_{2}\left(\mathbf{v}_{2}, \mathbf{j}_{2}\right)$. All cross section unit is Bohr length.
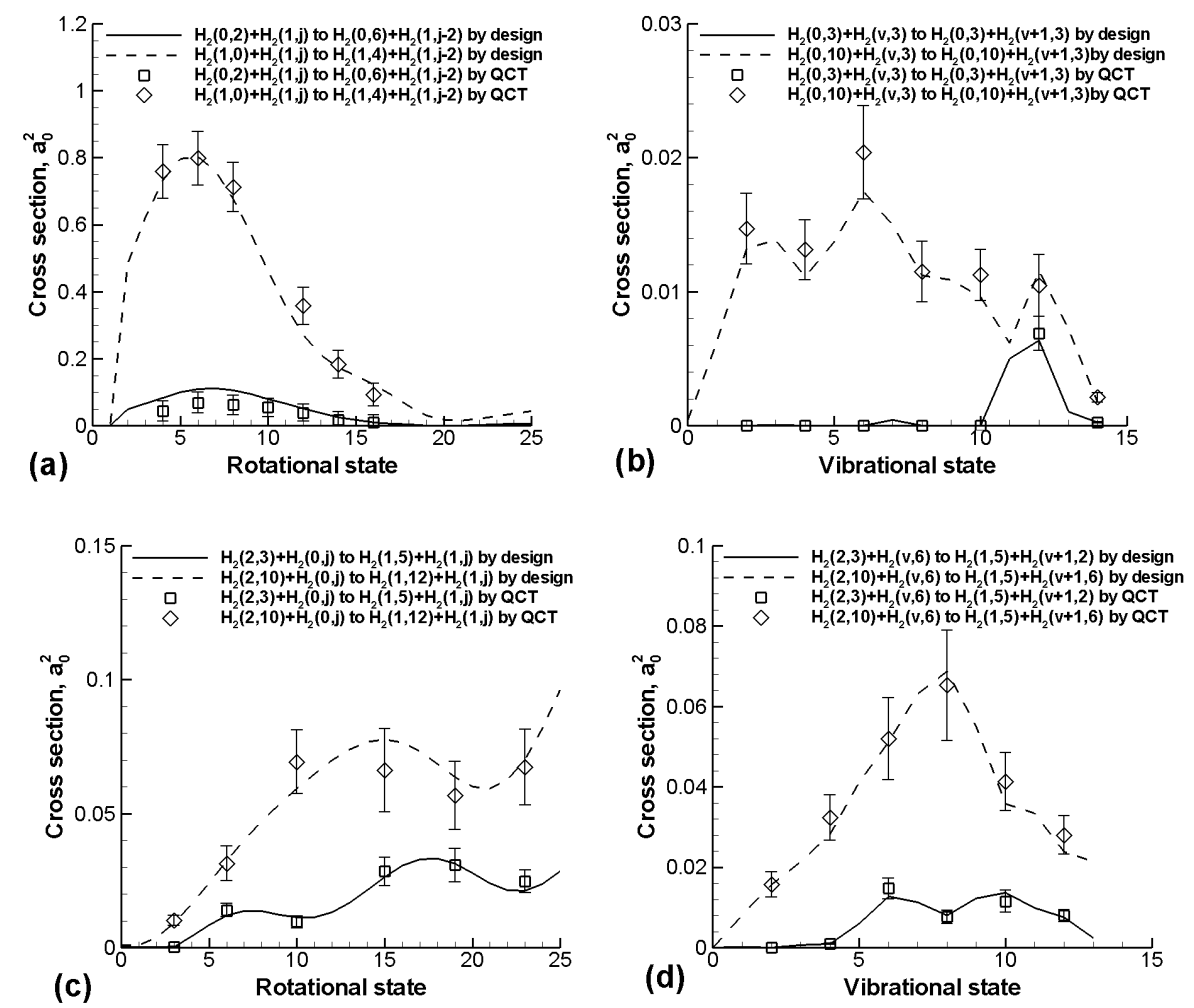

Figure 2. Comparisons of the state-to-state cross sections between the cross sections derived from the predicted response surface designed by the Kriging model and the cross sections calculated by QCT method (unused for the response surface design). 


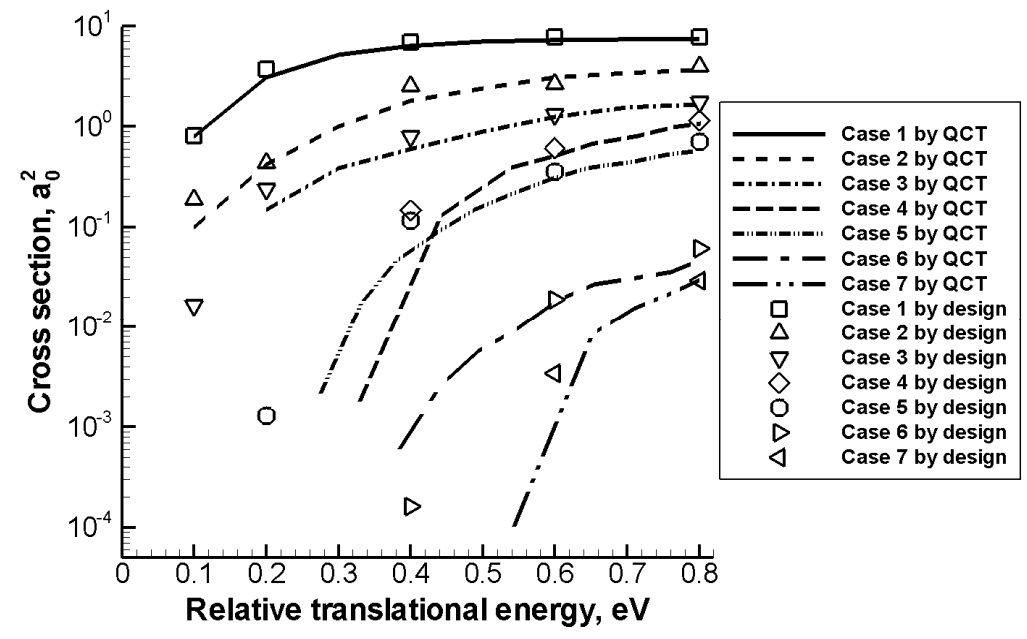

Figure 3. Comparisons of the state-to-state cross sections between the predicted response surface designed by the kriging model and the QCT calculations by Mandy and Pogrebnya, ${ }^{13}$ where case 1 is $\mathrm{H}_{2}(\mathbf{0 , 0})+\mathrm{H}_{2}(1,0) \rightarrow \mathrm{H}_{2}$ $(0,0)+H_{2}(1,2)$, case 2 is $H_{2}(0,2)+H_{2}(1,2) \rightarrow H_{2}(0,2)+H_{2}(1,4)$, case 3 is $H_{2}(0,2)+H_{2}(1,2) \rightarrow H_{2}(0,4)+H_{2}(1,2)$, case 4 is $\mathrm{H}_{2}(0,0)+\mathrm{H}_{2}(1,0) \rightarrow \mathrm{H}_{2}(0,2)+\mathrm{H}_{2}(1,2)$, case 5 is $\mathrm{H}_{2}(0,2)+\mathrm{H}_{2}(1,2) \rightarrow \mathrm{H}_{2}(0,4)+\mathrm{H}_{2}(1,4)$, case 6 is $\mathrm{H}_{2}(0,0)+\mathrm{H}_{2}(1,4) \rightarrow \mathrm{H}_{2}(0$ $, 2)+\mathrm{H}_{2}(1,0)$, and case 7 is $\mathrm{H}_{2}(\mathbf{0 , 0})+\mathrm{H}_{2}(1,4) \rightarrow \mathrm{H}_{2}(0,4)+\mathrm{H}_{2}(1,2)$.

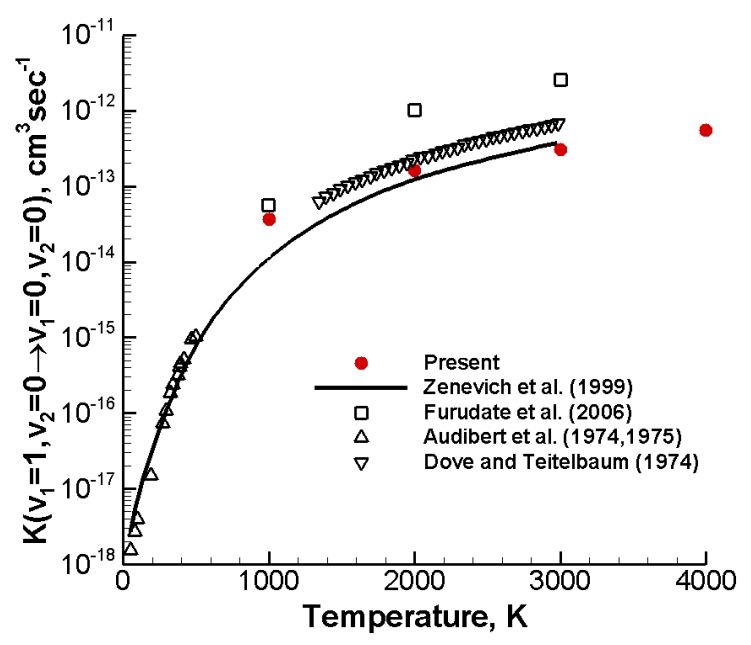

Figure 4. Comparisons of the present vibrational transition rates for para- $\mathrm{H}_{2}$ with the transition rates of the theoretical calculations ${ }^{15,16}$ and experimental measurements. ${ }^{17-19}$ 

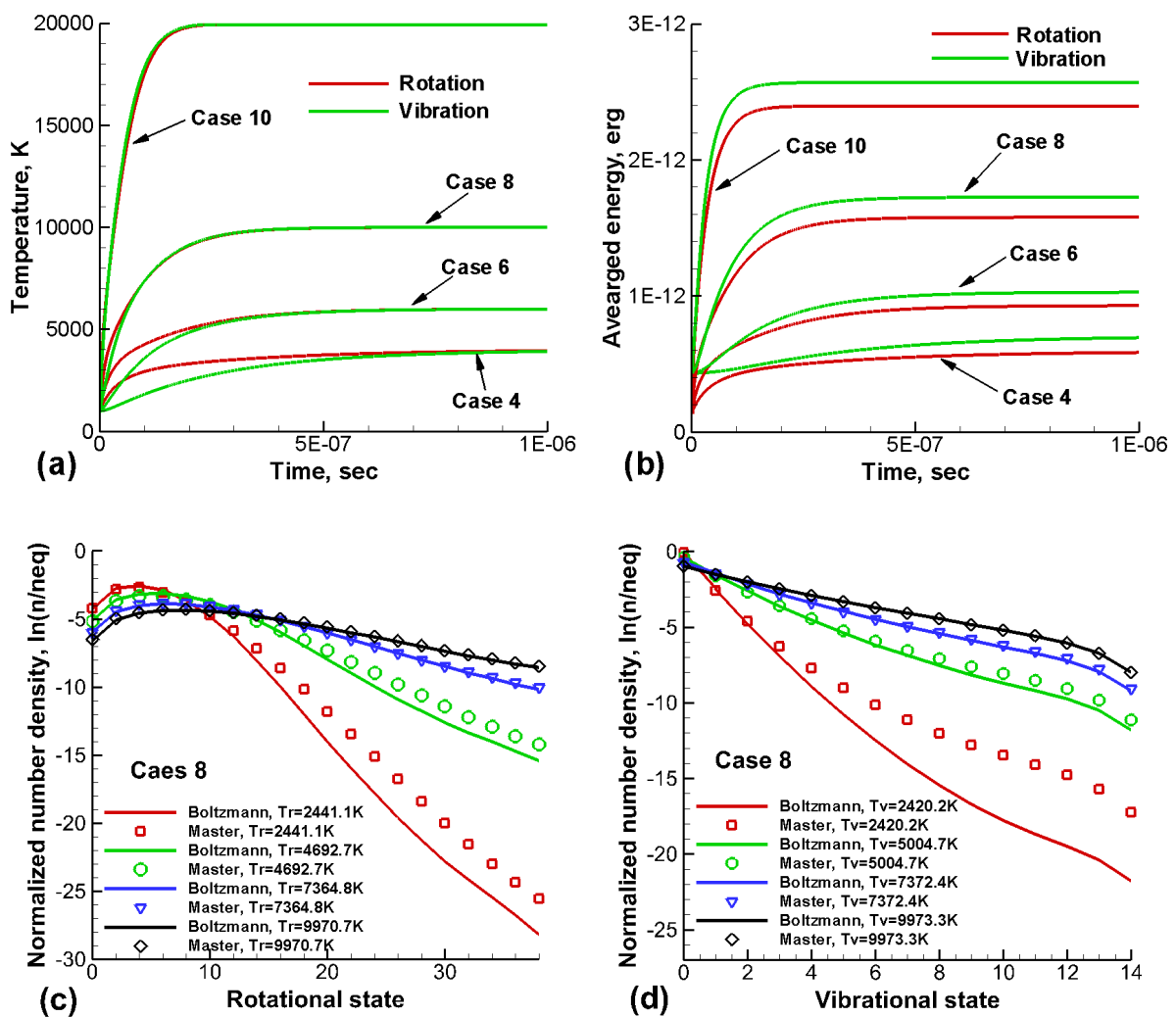

Figure 5. Relaxations of temperatures and number densities: (a) rotational and vibrational temperature relaxations, (b) averaged rotational and vibrational energy relaxations, (c) normalized number density distributions of rotation mode, and (c) normalized number density distributions of vibration mode.

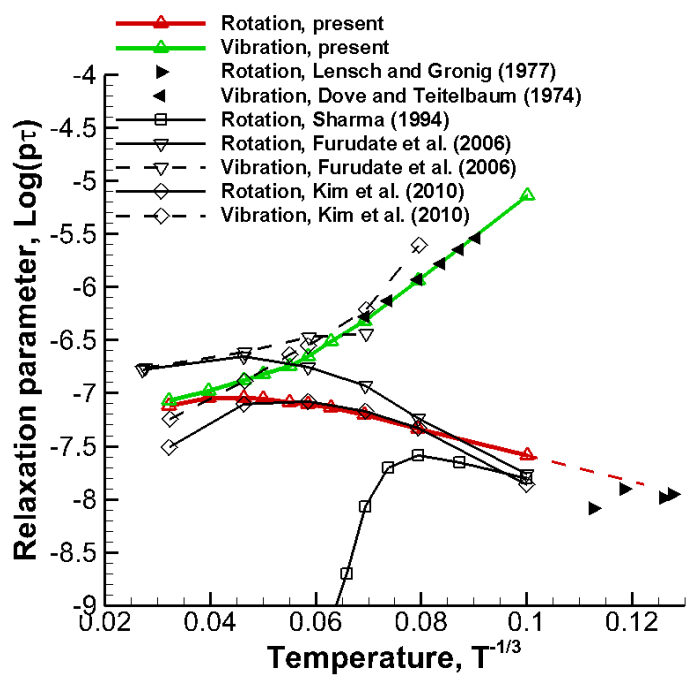

Figure 6. Comparisons of the present rotational and vibrational relaxation parameters with the experiments ${ }_{19,20}$ and theoretical calculations. ${ }^{16,21}$ 

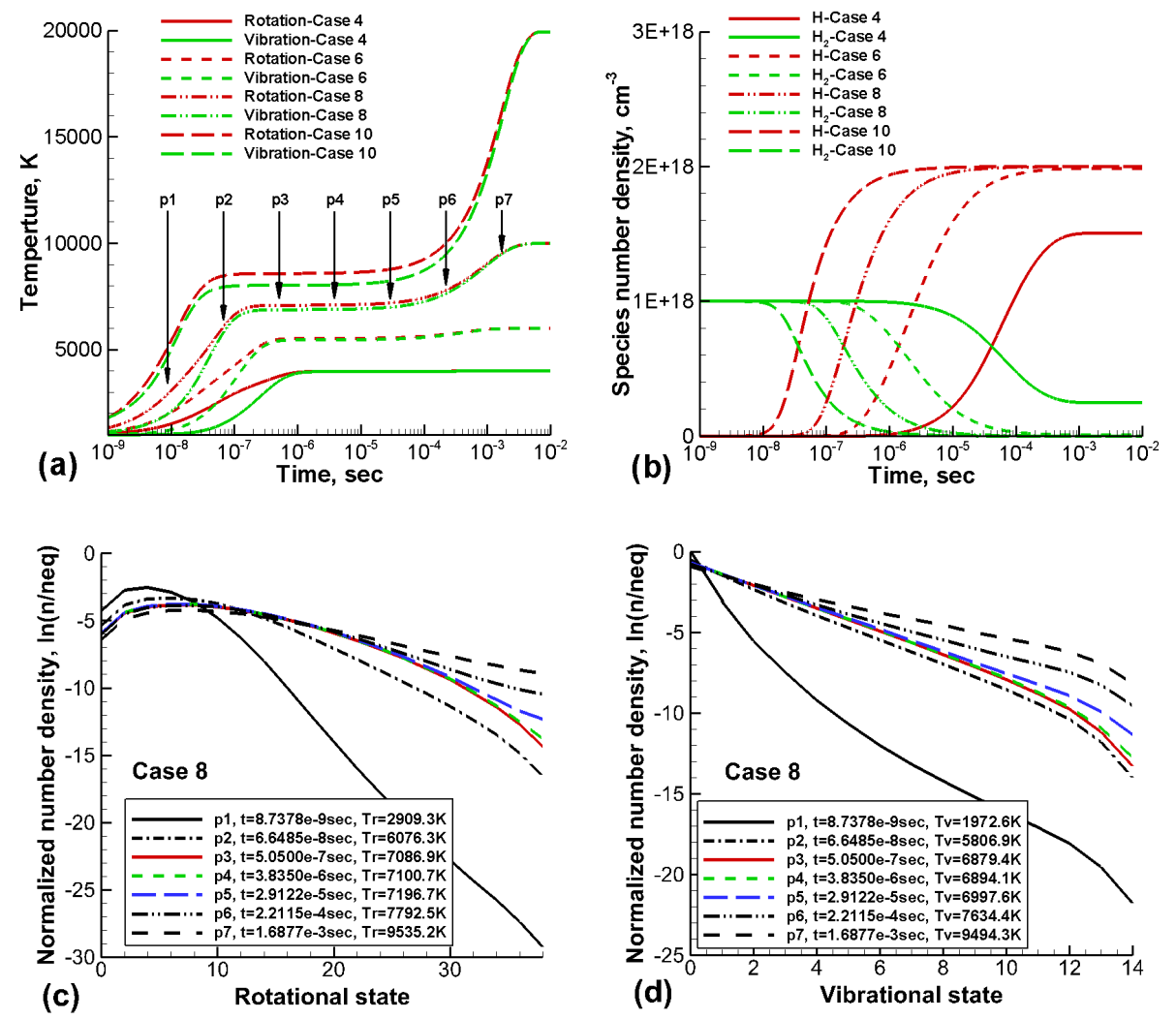

Figure 7. Relaxations of temperatures and number densities in heating cases: (a) rotational and vibrational temperature relaxations, (b) species number density relaxations, (c) normalized number density distributions of rotation mode, and (c) normalized number density distributions of vibration mode. 

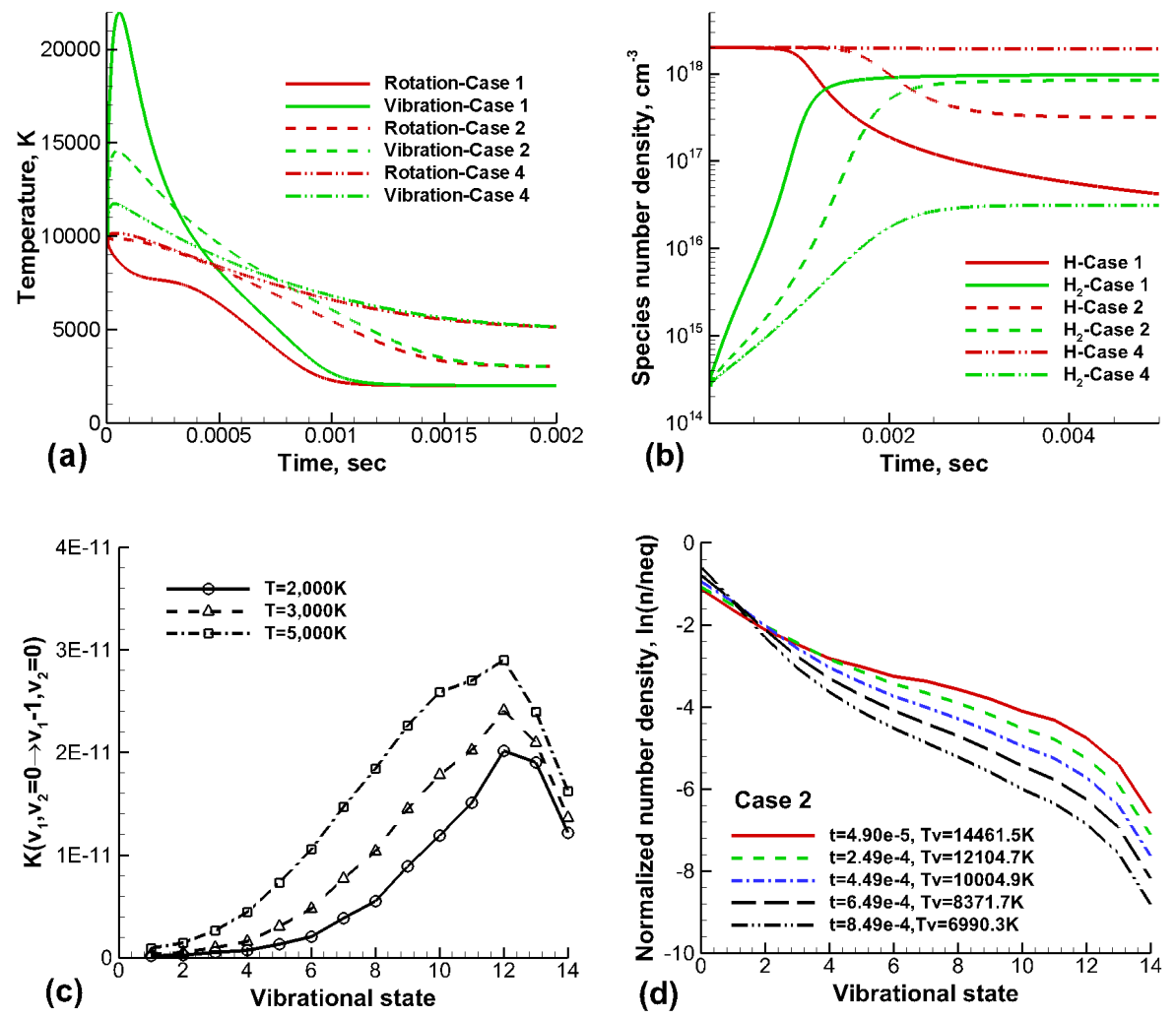

Figure 8. Relaxations of temperatures and number densities in cooling cases: (a) rotational and vibrational temperature relaxations, (b) species number density relaxations, (c) averaged transition rate coefficients for vibrational relaxation, and (c) normalized number density distributions of vibration mode.
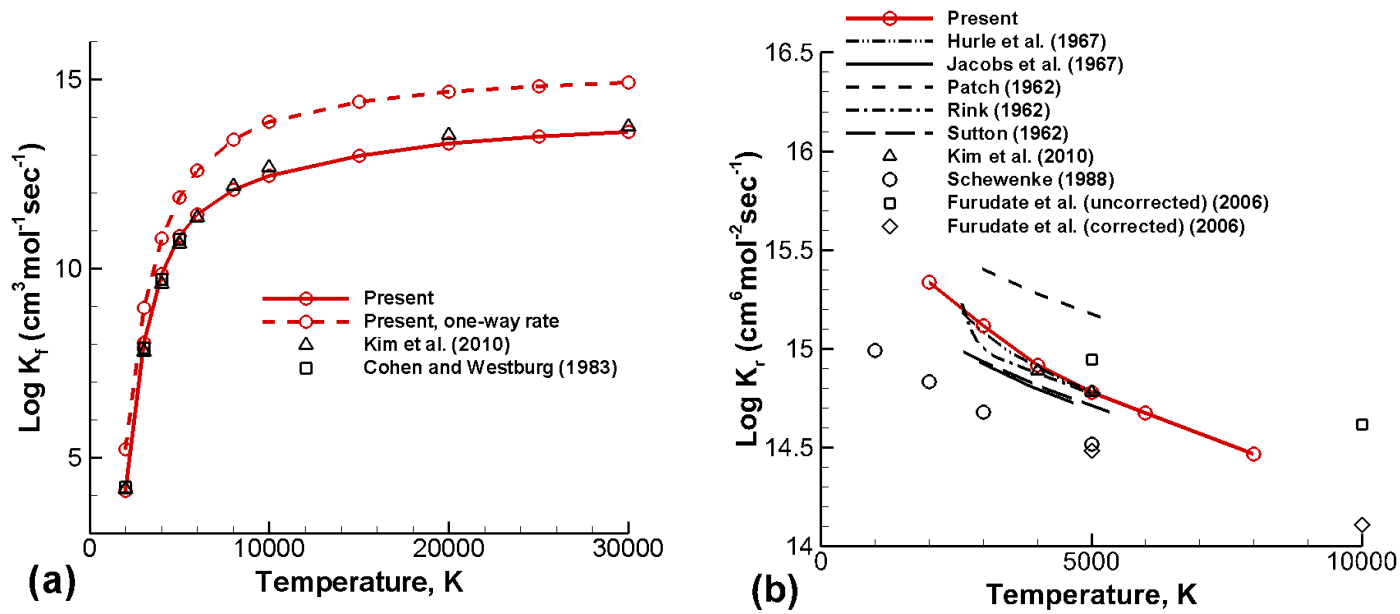

Figure 9. Comparisons of the present reaction rate coefficients with the experiments ${ }^{27-32}$ and theoretical calculations ${ }^{2,10,16}$ for dissociation and recombination reactions. 


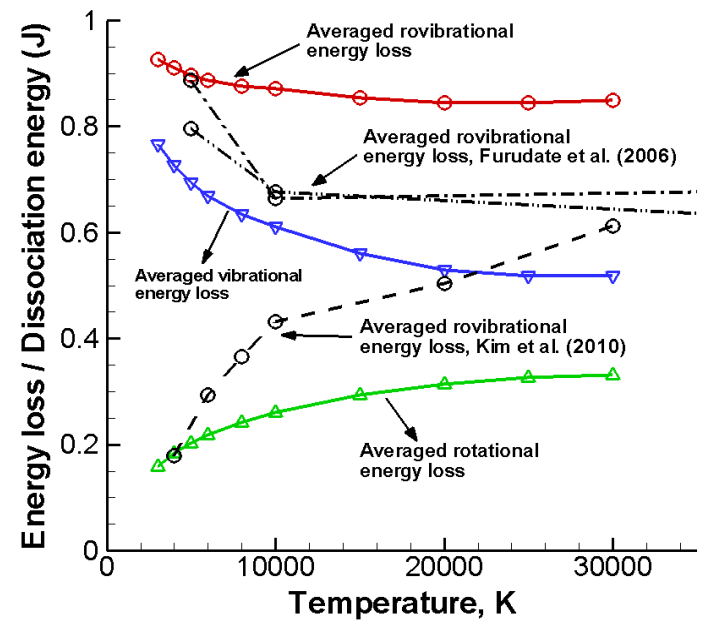

Figure 10. Comparisons of the rotational and vibrational energy losses between the present results and the previous theoretical calculations.
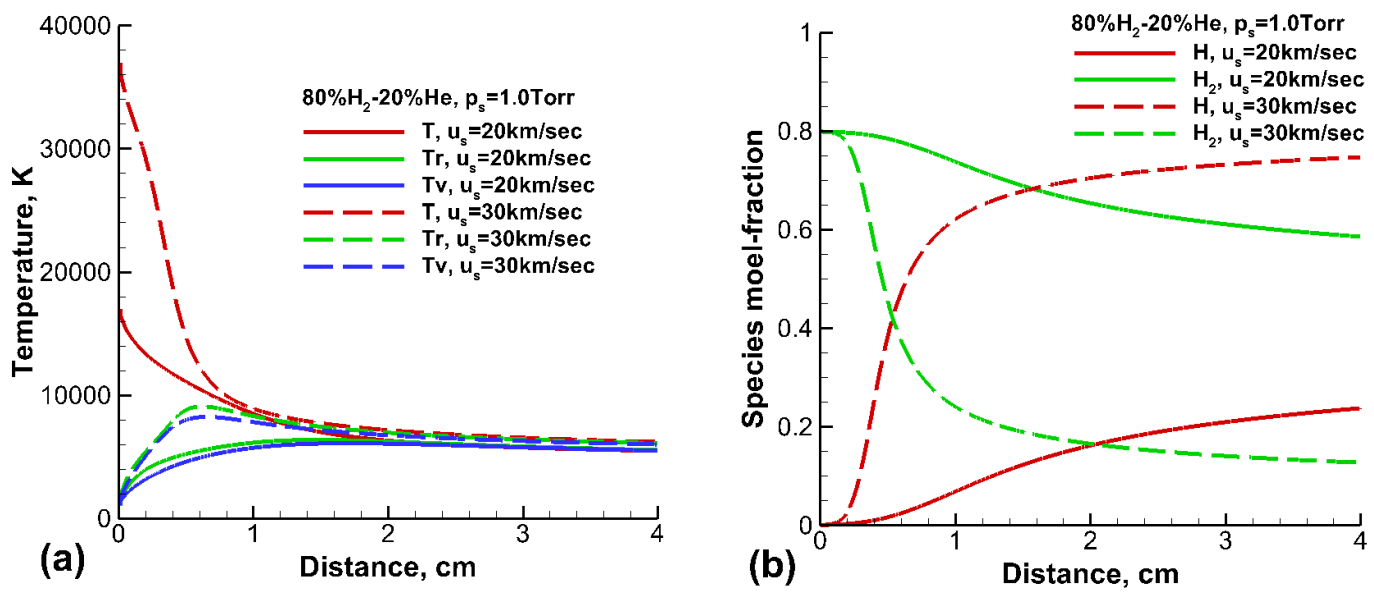

Figure 11. Temperatures and species mole-fractions in the post-normal shock flows 

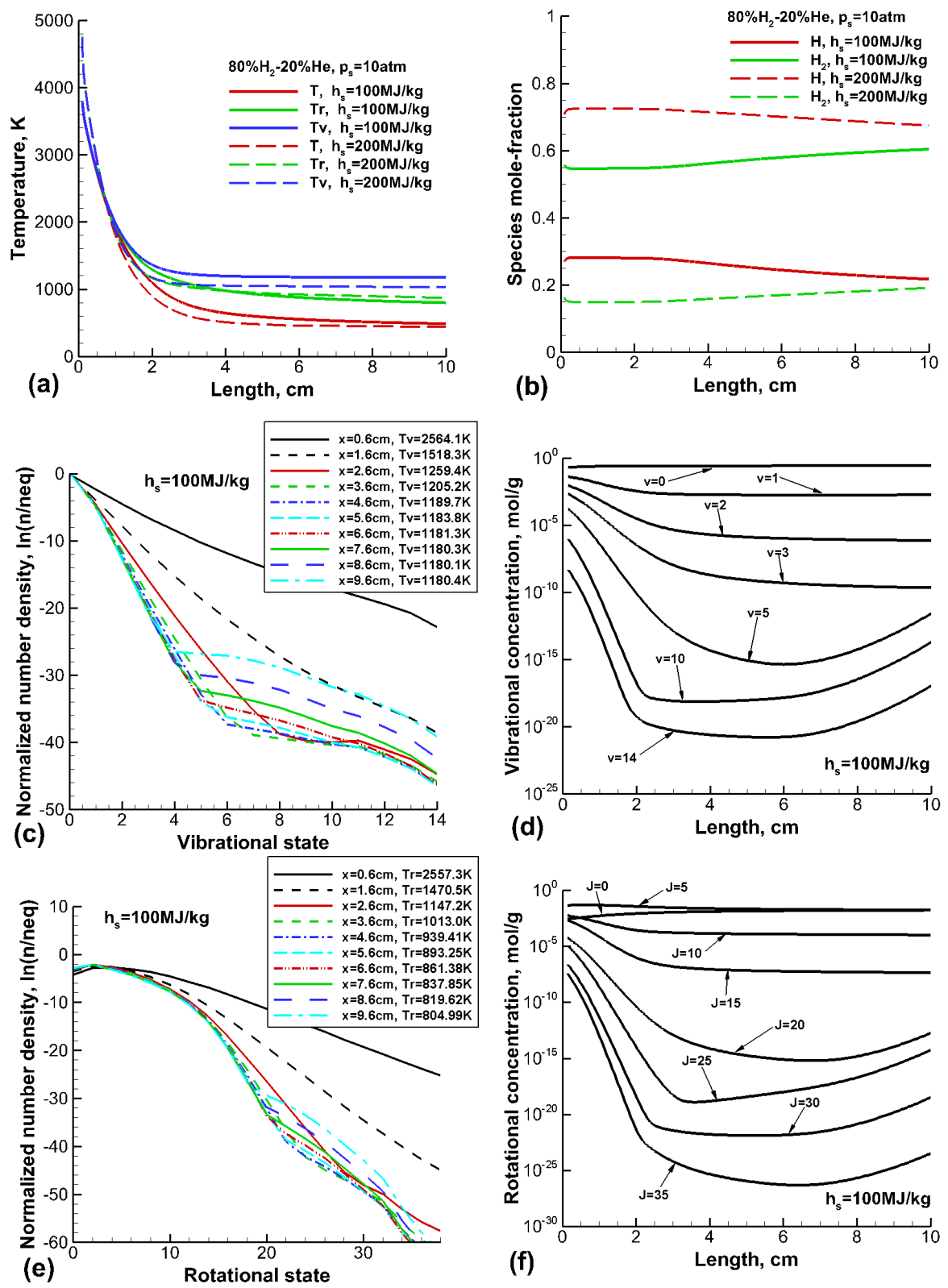

Figure 12. Temperatures, species mole-fractionsions, and number density distributions in the nozzle expanding flows: (a) relaxations of translational, rotational and vibrational temperatures, (b) relaxations of species mole-fractions, (c) normalized number density distributions of vibration mode, (d) relaxations of vibrational concentrations, (e) normalized number density distributions of rotation mode, and (f) relaxations of rotational concentrations. 